\title{
On TTEthernet for Integrated Fault-Tolerant Spacecraft Networks
}

\author{
Andrew Loveless* \\ NASA Johnson Space Center, Houston, TX, r7058
}

\begin{abstract}
There has recently been a push for adopting integrated modular avionics (IMA) principles in designing spacecraft architectures. This consolidation of multiple vehicle functions to shared computing platforms can significantly reduce spacecraft cost, weight, and design complexity. Ethernet technology is attractive for inclusion in more integrated avionic systems due to its high speed, flexibility, and the availability of inexpensive commercial off-the-shelf (COTS) components. Furthermore, Ethernet can be augmented with a variety of quality of service (QoS) enhancements that enable its use for transmitting critical data. TTEthernet introduces a decentralized clock synchronization paradigm enabling the use of time-triggered Ethernet messaging appropriate for hard real-time applications. TTEthernet can also provide two forms of event-driven communication, therefore accommodating the full spectrum of traffic criticality levels required in IMA architectures. This paper explores the application of TTEthernet technology to future IMA spacecraft architectures as part of the Avionics and Software (A\&S) project chartered by NASA's Advanced Exploration Systems (AES) program.
\end{abstract}

\section{Nomenclature}

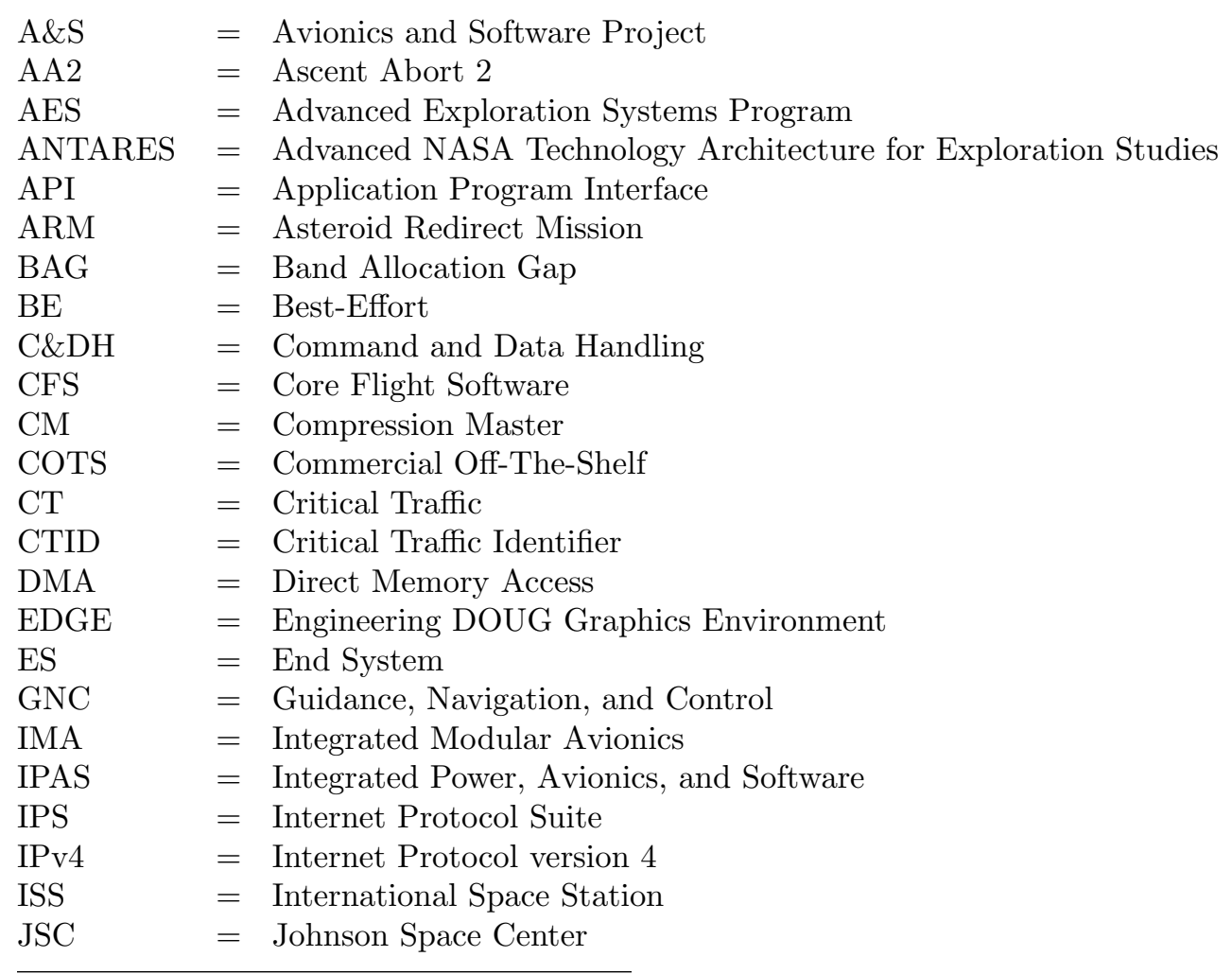

*Software Engineer, Command and Data Handling Branch, 2101 NASA Parkway, Houston, TX 


$\begin{array}{ll}\text { LAN } & =\text { Local Area Network } \\ \text { LAS } & =\text { Launch Abort System } \\ \text { MAC } & =\text { Media Access Control } \\ \text { MPCV } & =\text { Multi-Purpose Crew Vehicle } \\ \text { PCF } & =\text { Protocol Control Frame } \\ \text { PIO } & =\text { Programmed Input/Output } \\ \text { QOS } & =\text { Quality of Service } \\ \text { RC } & =\text { Rate-Constrained } \\ \text { RTOS } & =\text { Real-Time Operating System } \\ \text { SC } & =\text { Synchronization Client } \\ \text { SLS } & =\text { Space Launch System } \\ \text { SM } & =\text { Synchronization Master } \\ \text { TCP } & =\text { Transmission Control Protocol } \\ \text { TDM } & =\text { Time-Division Multiplexing } \\ \text { TT } & =\text { Time-Triggered } \\ \text { UDP } & =\text { User Datagram Protocol } \\ \text { VL } & =\text { Virtual Link } \\ \text { VOQ } & =\text { Virtual Output Queue }\end{array}$

\section{Introduction}

Aerospace projects have traditionally employed federated avionics architectures, in which each computer system is designed to perform one specific function (e.g. navigation). There are obvious downsides to this approach, including excessive weight (from so much computing hardware), and inefficient processor utilization (since modern processors are capable of performing multiple tasks). There has therefore been a push for integrated modular avionics (IMA), in which common computing platforms can be leveraged for different functions. ${ }^{1}$ One major challenge with this approach is the need to prevent non-critical failures from propagating through shared hardware resources and negatively impacting more critical systems. In fact, most avionic system failures result from ineffective fault containment and the resulting domino effect. ${ }^{2}$

As the glue connecting integrated systems, the data network plays a crucial role in error prevention and failure recovery. ${ }^{2}$ Furthermore, the network must accommodate traffic of mixed criticality and performance levels - potentially all related to the same shared computer hardware. The difficulty this poses causes most modern spacecraft to take a hybrid architecture approach that is neither fully federated nor integrated. Several different types of networks are incorporated, each suited to support a specific vehicle function.

Critical functions are typically driven by precise timing loops, requiring networks with strict guarantees regarding message latency (i.e. determinism) and fault-tolerance. ${ }^{3}$ One technique such networks employ is time-division multiplexing (TDM), in which messages are sent among connected nodes one at a time and in a specific order. This methodology reduces wiring by allowing network resources to be shared without conflicts. Early approaches to TDM in avionics, such as the MIL-STD-1553 data bus, used an asynchronous masterslave architecture. The need for central bus controllers to command remote devices (when to send/receive), however, resulted in low speeds and high overhead. ${ }^{4}$ Several more contemporary technologies instead follow a distributed time-triggered paradigm, in which clock synchronization between nodes enables precise scheduledriven communication. These architectures offer many advantages in safety-critical applications, lowering jitter while providing additional fault tolerance by removing single points of failure. ${ }^{5}$

Alternatively, non-critical systems generally employ data networks prioritizing flexibility and high performance over reliable operation. Examples of these technologies are often found interfacing communication equipment and high speed instrumentation to onboard computer systems. The success of switched Ethernet technology in filling this role in terrestrial applications makes it desirable for inclusion in future spacecraft platforms. Basic Ethernet configurations have been incorporated into several preexisting aerospace projects, including both the Space Shuttle and International Space Station (ISS). ${ }^{6}$

But Ethernet's use has not been limited to handling noncritical data. Over the past several decades, many proprietary Ethernet variants (with various quality of service (QoS) enhancements) have been created for time-critical industrial and manufacturing applications. This push for "Industrial Ethernet" largely stems from its scalability, high speed, and the availability of inexpensive commercial off-the-shelf (COTS) components. Similarly, ARINC 664-p7 standardizes an Ethernet variant with increased determinism and 
reliability for aerospace applications. ${ }^{7}$ It accomplishes this through the concept of rate-constrained traffic, in which predetermined knowledge of traffic patterns can ensure a theoretical upper bound on transmission delays.

TTEthernet ${ }^{a}$ further closes the gap towards the goal of achieving fully deterministic Ethernet communication by introducing the principles of decentralized clock synchronization and time-triggered messaging. Besides time-triggered messages (TT), TTEthernet is also capable of supporting two forms of event-driven communication - rate-constrained (RC) and best-effort (BE). The RC traffic class is functionally equivalent to ARINC 664-p7. BE traffic behaves comparably to traditional layer 2 Ethernet, with no guarantees regarding timely (or even successful) message delivery. ${ }^{8}$ In this way, TTEthernet overcomes difficulties in realizing an IMA architecture, providing three distinct traffic classes covering the entire spectrum of criticality levels. Common computing platforms (e.g. line-replaceable units) can share networking resources in such a way that failures in non-critical systems (using $\mathrm{BE}$ or RC communication modes) cannot impact flight-critical functions (using TT communication). A variant of TTEthernet, named Time-Triggered Gigabit Ethernet (TT-GbE), is deployed as part of the onboard data network (ODN) on NASA's Orion spacecraft.

This paper explores the application of the TTEthernet technology to future spacecraft as part of the Avionics and Software (A\&S) project chartered by NASA's Advanced Exploration Systems (AES) program. First, goals of the A\&S project are stated, and IMA considerations in network development are presented. The shortcomings of classical Ethernet in time-critical applications are discussed, along with how the TTEthernet technology overcomes these deficiencies. Next, a general overview of TTEthernet is provided. The use of TTEthernet is then demonstrated, providing real-time failover capability between redundant flight computers running Core Flight Software (CFS) in two distinct simulated mission scenarios: 1) Asteroid Redirect Mission (ARM) and 2) Orion Ascent Abort 2 (AA2) flight test. The AA2 mission in particular imposed significant constraints on the flight control loop - necessitating the development of a software-level network stack to accommodate larger data payloads, as well as the implementation of network-based flight computer synchronization to handle failure in such a critical mission phase. Lastly, the application of TTEthernet to future projects is discussed.

\section{The Avionics and Software Project}

As private industry begins transporting astronauts to the International Space Station (ISS) through the Commercial Crew Program, NASA will shift its focus towards furthering human spaceflight exploration beyond low earth orbit (LEO). Though future mission scenarios are unknown, possible targets include EarthMoon Lagrange points, near earth asteroids (NEAs), and Mars. ${ }^{9}$ Though vehicle requirements differ for each objective, spacecraft avionics must incorporate similar technologies to enable success in any deep space mission. Because the field of avionics is advancing so rapidly in terrestrial applications, NASA can minimize development time and cost by utilizing existing commercial technologies.

It is the goal of the A\&S project to discern the most promising of these options, mature them for future use, and lower the risk of their inclusion in other projects. Leveraging this work, the A\&S project is developing a flexible mission agnostic spacecraft architecture according to IMA principles that is adaptable to a wide variety of future mission scenarios. The A\&S team includes participants across NASA centers, other government agencies, and industry partners. The Integrated Power, Avionics, and Software (IPAS) facility at Johnson Space Center (JSC) is used extensively to demonstrate integration, providing a flexible evaluation environment for avionics components and software in a realistic framework of vehicle subsystems. ${ }^{6}$

\section{IMA Considerations in Networking}

Probable future missions (e.g. NEAs, Mars) may take months to years to complete. However, while long term missions operating in LEO can be periodically resupplied from earth, it is too costly to continuously support vehicles traveling to further destinations. ${ }^{9}$ This combination of long duration and inaccessibility highlights the importance of adopting integrated modular avionics principles when designing future spacecraft architectures.

Early manned spacecraft, such as those of the Apollo program, were designed with federated architectures, in which no distinction exists between software and hardware. Each computer system was built to perform

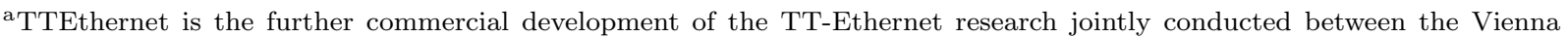
University of Technology and TTTech Computertechnik AG. ${ }^{8}$
} 
one specific function. Because software under this paradigm is inherently contained by distinct hardware platforms, faults cannot propagate to other systems. Space partitioning also ensures that software need not compete for processor resources. ${ }^{1,2}$ Finally, the point-to-point wiring used in such architectures is highly deterministic.

Though the federated methodology looks good on paper, it has several drawbacks - especially within the context of vehicles designed for long duration deep space missions. As many functions exist on a spacecraft, the need for so many computing platforms results in excessive weight and power consumption. This effect is compounded by the duplication of critical components (needed for redundancy) and the large number of unique spares required to rectify failures. Additionally, long heavy runs of cable are necessary to connect the vehicle's many discrete systems. The contribution of cabling to total vehicle weight cannot be ignored. In fact, the American Institute of Aeronautics and Astronautics (AIAA) recommends that 5-8\% of a vehicle's on-orbit dry mass budget be allocated for cables alone. ${ }^{10}$

All these drawbacks present major issues when considering future spacecraft with limited ground support and likely severe volume and mass restrictions. Also, the sheer amount of processors, spares, and wiring needed to realize a federated architecture significantly increases the cost of avionic systems, which in turn can comprise more than $50 \%$ the cost of aerospace projects. ${ }^{11}$ Moreover, because each system is custom built to serve one function, any change to the vehicle architecture (e.g. networking previously unconnected devices) necessitates expensive hardware modification.

The IMA approach to avionics design emphasizes the use of shared hardware that performs multiple distinct vehicle functions. This methodology has been made possible by radical advancements in computing technology over the last several decades. Modern processors are more than capable of handling multiple concurrent tasks, and faults can still be contained through techniques such as space partitioning (e.g. dedicated registers or I/O) and software fault isolation. ${ }^{2}$ IMA offers significant cost and weight savings over federated techniques, as the amount of computing platforms, spares, and wiring can be drastically reduced. Additionally, IMA's emphasis on interchangeable components significantly increases overall system maintainability by enabling one kind of spare to correct multiple types of failures. Proper use of IMA principles can also significantly simplify the development process. The use of standard interfaces allows the vehicle's architecture to adapt as its requirements evolve.

The main objective of an integrated architecture is to consolidate spacecraft functions to shared hardware resources, while keeping the reliability federated designs provide. Accomplishing this task requires a "jack of all trades" data network, capable of accommodating traffic from multiple highly diverse systems (e.g. critical vs non-critical) that may all be realized on one shared computer platform. Because individual network technologies are rarely so competent, however, the adoption of true IMA principles to network architectures often proves unreasonable. Applying the same network architecture to the entire spacecraft, and thus systems of all criticality levels, generally results in undue expense and limited performance.

To illustrate this point, first imagine the communication architecture needed to stream 1080p video from a vehicle's external cameras to an onboard crew display. What traits must this network possess? High throughput is obviously necessary, along with the flexibility to expand the network with new cameras as needed. Next consider the network required to relay real-time measurements from a spacecraft's external proximity sensors to the flight computers. Could the video network also be leveraged to transmit this sensor data? Likely not, as it was not designed to guarantee the constant latency required by such a system.

Another major concern with consolidated networks is the potential for cascading faults between two systems utilizing the same physical network connections. For example, it may be possible for a video camera to malfunction (e.g. babbling idiot behavior) saturating the network and preventing the delivery of the much more critical sensor readings. The difficulty of implementing a truly integrated network causes most modern spacecraft architectures to take a hybrid approach, including four or more network technologies in one vehicle. For example, NASA's lunar reconnaissance orbiter (LRO), launched in 2009, employs a MILSTD-1553 bus, a SpaceWire network, high speed RS-422 lines, and multiple low-voltage differential signaling (LVDS) interfaces. ${ }^{12}$

\section{Ethernet is Promising}

Ethernet technology has advanced tremendously over the last few decades, becoming a promising network candidate in the shift to more integrated systems. Ethernet is a family of standardized networking technologies for peer-to-peer communication between nodes within a local area network. Originally developed at 
Xerox PARC in the early 1970s, Ethernet quickly overtook competitors to become the dominant local area network (LAN) technology in the business and IT sectors. Early implementations utilized a bus topology in which multiple devices connect to one shared cable medium. In this case, an access control method is needed to regulate bus traffic and limit message collisions. One such method, Carrier Sense Multiple Access with Collision Detection (CSMA/CD), directs nodes to wait a random interval after a collision is detected before messages can be resent. ${ }^{13}$ Though a passable solution for low traffic networks, this behavior results in unacceptable delays as network utilization rises (and collisions become unavoidable). Switched Ethernet introduced full duplex operation, consequently eliminating the problem of collision by allowing each node to send and receive packets simultaneously. Ethernet has become ubiquitous in day-to-day life and now presents a viable option for integrated avionics architectures.

Ethernet's pervasive nature makes ensuring interoperability among devices easy to accomplish. Almost all hardware required to transmit or receive information, from gyroscopes to power controllers, can communicate using Ethernet either natively or through the use of inexpensive adapters. The widespread availability of affordable compatible COTS Ethernet products is one of its most prominent advantages. Because COTS components have already been matured to the point of successful operation, minimal additional development is necessary before adoption - reducing cost and removing the schedule risk associated with the development of custom electronics. The high degree of commonality between Ethernet hardware also promotes interchangeability between network devices and connectors. Furthermore, Ethernet networks are easily expanded, permitting the addition of new devices without any expensive hardware modification or changes to the network structure itself. As the architecture evolves, applications can be adapted using well-defined application program interfaces (APIs) and programming practices. Similarly, code can be recycled from previous projects to meet new requirements.

All these traits are highly desirable in a network required to integrate multiple systems. Additionally, Ethernet's high throughput expands the range of traffic for which it can be utilized, even accommodating high speed data streaming beyond the capabilities of most competing network technologies. Perhaps most importantly, Ethernet can be augmented with a variety of QoS enhancements that enable its use for transmitting critical data in hard real-time applications. Ethernet technology and its variants therefore permit the creation of an integrated network architecture covering the full spectrum of traffic criticality levels.

Through consideration of the above factors, the A\&S project has concluded that Ethernet is fundamental in the design of any future avionics architecture for manned spacecraft. The utilization of Ethernet technology has also been encouraged by the A\&S project's decision to maximize the use of Core Flight Software (CFS) whenever possible. Certified for human spaceflight at Johnson Space Center in 2014, CFS provides a set of flexible software services compatible with a variety of processor architectures and operating systems. In CFS, separate applications each perform distinct functions on the vehicle - including controlling power data units (PDUs) and monitoring cabin pressure. These applications all heavily employ the Internet Protocol Suite (IPS) for onboard communication, further supporting the need for Ethernet in future vehicle architectures. The A\&S project's focus on Ethernet communication, and utilization of Core Flight Software, has led to the development of a well-defined IP-based backbone showcased in Johnson Space Center's IPAS facility. This infrastructure, however, is only suitable for transmitting nonessential data with low quality of service requirements. ${ }^{6}$

\section{The Shortcomings of Classical Ethernet}

Though the introduction of switches stopped collisions, timing within an Ethernet network is still not predictable. This shortcoming stems from the event-driven nature of its communication, in which messages are only sent in response to environmental or internal events. To understand how this variable delay occurs, it is first necessary to explore the underlying fundamentals of switch operation.

The basic purpose of a network switch is to forward frames from a given input (ingress) port to a particular output (egress) port. Modern switches accomplish this task through a crosspoint matrix switching fabric. ${ }^{13}$ The event-triggered paradigm of Ethernet communication suggests that multiple messages will, at some point, inevitably need to travel through this matrix simultaneously. This concurrent communication is usually supported by nature of the switch fabric's parallel arrangement, in which data pathways are separated through space partitioning. Collisions, however, eventually occur as multiple frames are simultaneously forwarded to the same output port. ${ }^{14}$

This phenomenon closely resembles the issue of message collision observed in early Ethernet systems 
utilizing shared bus technology. As CSMA/CD was employed to regulate access to a shared data medium, crossbar switches must too utilize a process to regulate input to the switch fabric. This regulation is governed by an onboard processor commonly referred to as the switch allocator. For each clock cycle, the switch allocator must determine which of the switch's ingress and egress ports should be connected. ${ }^{15}$ Several schemes exist to provide fair contention resolution at high rates.

Simple approaches employ queuing buffers at the incoming switch ports. This memory space is managed by the switch allocator and used to temporarily store messages entering the switch. As messages are received, the input buffers send requests to the switch allocator for connections to the output ports based on the destinations of the frames they contain (determined by routing logic). In an arrangement employing a single first-in, first-out (FIFO) queue per switch input, each ingress queue may make one request per clock cycle. A simplified switch allocator is composed of one fabric arbiter per output channel. Each arbiter recognizes when multiple buffers are requesting access to its output port. To prevent collision, it uses an arbitration scheme to decide which input port to service. Each arbiter performs this operation independently, and may only grant one request per clock cycle. ${ }^{14,15}$

This collision arbitration process makes transmission time in Ethernet networks inherently unpredictable. The delay a message experiences when passing through a switch depends heavily on contention from competing inputs and the arbitration method used. Since messages may reach the network switches at any time, the frequency and severity of conflicts is highly variable.

Contention within the switch can significantly limit its overall throughput. In fact, basic switches employing input FIFO queues can realistically only exhibit a maximum throughput of $58.6 \%$ under uniform traffic arrivals. ${ }^{16}$ In extreme cases, the switch may not be able to keep up with the rate at which it receives frames, the internal buffers may overflow, and messages must be discarded. Higher level protocols (e.g. Transmission Control Protocol (TCP)) can ensure successful delivery and message sequencing, but introduce significant overhead and unpredictable delay from repeated transmissions.

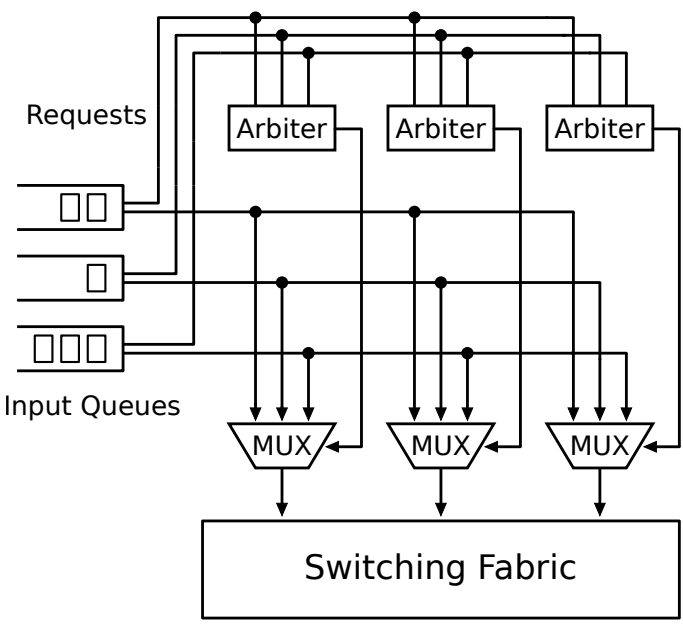

Figure 1. Input-buffered switch described in Ref. 15.

Efforts to increase throughput in network switches include the use of virtual output queues (VOQs), crosspoint buffers, and improved arbitration procedures (matrix, wavefront). The use of VOQs particularly improves throughput in modern high performance switches, lowering congestion by having each input port maintain a separate queue for each possible output. Because each VOQ at a given input port corresponds to a separate output channel, frames intended for busy output ports do not prevent messages destined for free ports from being delivered. In this way, use of VOQs can eliminate the classical head-of-line blocking problem seen in basic FIFO input-buffered switches. ${ }^{15}$ Switches employing VOQs can achieve $100 \%$ throughput under uniform traffic, but not under more realistic non-uniform traffic patterns. Two-stage switches have been proposed to overcome this barrier. The first stage acts as a load balancer, spreading traffic evenly over the second stage - a typical VOQ input-buffered switch. Such designs have achieved $100 \%$ throughput in simulation, but introduce additional complexity as packets may be arbitrarily mis-sequenced between stages. ${ }^{17}$

Even with the addition of VOQs, the amount of delay experienced by any one frame is highly variable. This behavior results from two major limitations: 1) only one VOQ per input port may forward a message per clock cycle, 2) output ports can only send one message per clock cycle. In the unpredictable case of multiple frames competing for access to the same egress port, transmission is delayed an unknown amount as messages are forwarded out one at a time. ${ }^{18}$

We see then that the uncoordinated nature of Ethernet communication, even with modern advancements in switch technology, unavoidably leads to message contention within network switches. In all such instances an arbitration process must be used to resolve conflicts, introducing variation in the time it takes for messages to be forwarded. Though well suited for some purposes, network technologies requiring an arbitration process for message transmission are commonly considered inadequate for use in hard real-time spacecraft applications. ${ }^{5}$ Even priority-based arbitration schemes, in which higher priority messages are expressly favored, do 
not provide tolerable certainty. Minor variations in message latency can alter which messages contend at any given arbitration cycle and quickly result in unanticipated system behavior. ${ }^{19}$ Flight critical functions must operate in an entirely predictable manner, and thus require a high level of network determinism that classical Ethernet cannot provide.

\section{The Role of TTEthernet in the A\&S Project}

The A\&S project's investigation of TTEthernet is an effort to extend the capabilities of the current Ethernet backbone to accommodate time-critical data, thus realizing a more closely integrated network architecture. TTEthernet can support the full spectrum of traffic criticality levels by employing eventbased and time-triggered traffic classes. These enable the simultaneous transmission of both high speed low-priority and time-critical data within the same network and over the same physical layer. ${ }^{8}$ In this way, TTEthernet solves one major problem faced when designing networks according to IMA principles - finding a network technology suitable for the full range of traffic varieties transmitted from a shared computer system performing functions with multiple criticality levels. Furthermore, TTEthernet's traffic classes provide a hard fault containment method within the network itself, as delivery of time-triggered messages is guaranteed regardless of erroneous traffic patterns in lower priority classes.

Fault containment, however, is not limited to the interaction between traffic classes. The scheduled nature of time-triggered messaging ensures that critical systems may only access the network at times previously specified in the communication schedule. Furthermore, switches may be configured as bus guardians to contain the arbitrary failure of individual TTEthernet devices, preventing well known faults likes babbling idiot behavior from affecting other systems. Cascading faults between computing platforms can thus be reduced without the need for complex fault isolation procedures within the software applications themselves.

Additionally, TTEthernet employs the same frame payload size and structure as classical Ethernet. An existing application using conventional Ethernet communication can therefore be modified to instead employ TTEthernet without requiring major software changes. This similar structure also enables the use of common higher level protocols, adding services like fragmentation/assembly, on top of TTEthernet's data link layer. The A\&S project is particularly attracted to the technology's compatibility with its existing Ethernet network architecture. TTEthernet hardware (e.g. switches, cabling) can be shared by both systems employing TTEthernet and classical Ethernet, saving weight, lowering power, and reducing integration complexity.

\section{TTEthernet Overview}

This section explores key aspects of the TTEthernet protocol. Its goal is to provide a detailed overview of the network structure, the role of each traffic class, and the synchronization process.

\section{VII.A. Network Structure}

TTEthernet networks are composed of specialized end systems and network switches connected through bidirectional copper or fiber Ethernet links. An end system is typically realized as a TTEthernet network adapter that occupies a standard expansion bus (e.g. CompactPCI, PCIe) in a computing platform. Alternatively, an end system can be implemented on a field-programmable gate array (FPGA) or even as a standalone software stack. Radiation hardened switches and end systems are also in development for future spacecraft missions beyond LEO.

Message transmission between end systems is conducted according to a time-triggered paradigm. A network planning tool is used to allocate each device a finite amount of time in which it may transmit a frame. Each time slot is repeated sequentially to form a periodic communication schedule that is then loaded onto each TTEthernet device (e.g. switches and end systems). Once the devices are configured, a decentralized synchronization process establishes a global time base among all network participants. The communication schedule on each component references this synchronized time in order to dispatch messages at predetermined instances. This schedule guarantees that no contention exists between time-triggered Ethernet frames in the network switches, therefore eliminating the need for arbitration (and the timing variation it causes). End-to-end latency can thus be kept extremely low, and most importantly, nearly constant (jitter $<1 \mu \mathrm{s}) .{ }^{20}$ Also, delivery of time-triggered messages is guaranteed.

Virtual links (VLs) are used to direct time-triggered messages between end systems. Each VL is defined 
during the scheduling process and designates a logical connection between one sender and one or more recipients. The virtual link associated with each time-triggered frame is denoted by a critical traffic identifier (CTID) occupying two bytes within the Ethernet header. The CTID is used to replace traditional media access control (MAC) based delivery seen in classical Ethernet systems. Each TTEthernet switch uses a statically defined forwarding table to associate individual VLs with corresponding output ports. All messages sent along the same virtual link are guaranteed to take the same path through the network as long as the network configuration remains unchanged. ${ }^{21}$ This predictable behavior helps ensure constant transmission time between nodes. Furthermore, increased fault-tolerance can be achieved by using one virtual link to send duplicated messages through multiple redundant switches.

Figure 2 demonstrates a sample TTEthernet network consisting of one switch and four end systems (ES1, ES2, ES3, ES4). Solid lines are used to represent physical connections, and dashed lines are used to represent data flow within the network. ES1 sends identical frames to both ES3 and ES4 through VL6. ES4 transmits data to ES2 through VL8.

Figure 3 shows the structure of a time-triggered Ethernet frame compared to that of a standard IEEE 802.3 Ethernet frame. In both cases, a 7 byte preamble allows the receiver to synchronize with the timing of the received data, while a 1 byte start frame delimiter (SFD) is used to indicate the start of a new frame. In a standard Ethernet frame, the next two 6 byte fields contain MAC addresses for both the destination and the source. The following 2 byte field is used to indicate either the length of the data payload or the EtherType of the encapsulated protocol. This field, along with the MAC address fields, composes the Ethernet header. The actual

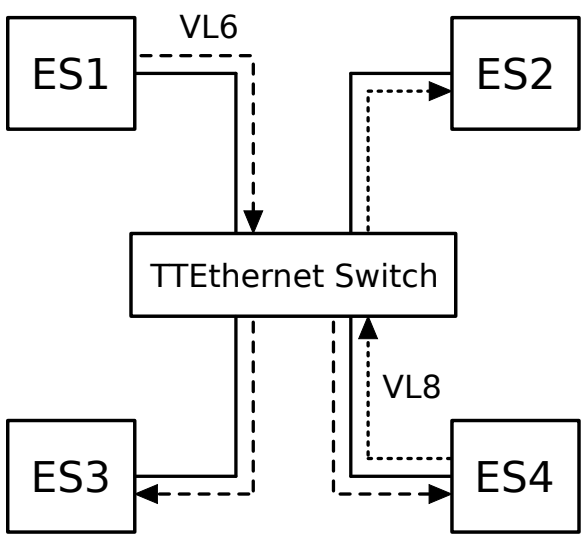

Figure 2. A sample TTEthernet network. data payload follows, occupying between 46 and 1500 bytes. Next, a 4 byte frame check sequence (FCS) is used for error detection. Lastly, an interpacket gap (IPG) equivalent to at least 12 bytes is required before transmission of a new frame..$^{22,23}$

\begin{tabular}{|c|c|c|c|c|c|c|c|}
\hline bytes & 1 byte & \multicolumn{1}{c}{6 bytes } & 6 bytes & 2 bytes & $46-1500$ bytes & 4 bytes \\
\hline Preamble & SFD & $\begin{array}{c}\text { Destination } \\
\text { Address }\end{array}$ & $\begin{array}{c}\text { Source } \\
\text { Address }\end{array}$ & $\begin{array}{c}\text { EtherType } \\
\text { (Length) }\end{array}$ & Data Payload & FCS & IPG \\
7 bytes & 1 byte 4 bytes 2 bytes & 6 bytes & 2 bytes & $46-1500$ bytes & 4 bytes 12 bytes \\
\hline Preamble & SFD & CT Marker & CTID & $\begin{array}{c}\text { Source } \\
\text { Address }\end{array}$ & Length & Data Payload & FCS \\
\hline
\end{tabular}

Figure 3. Structure of 802.3 Ethernet (top) and Time-Triggered Ethernet (bottom) frames (layer 2 only).

The only changes necessary to transform a standard Ethernet frame into a time-triggered frame (with no encapsulated protocol) are within the Ethernet header. In place of the MAC destination field is a 4 byte CT Marker and a 2 byte CTID. The CT Marker is a static identifier used to distinguish time-triggered frames from other Ethernet traffic. The CTID is used by the switches to route time-triggered frames through the network. Finally, the last 2 bytes of the header are expressly used to contain the payload length.

\section{VII.B. Traffic Class Integration}

Besides time-triggered messaging, TTEthernet networks may provide two additional traffic classes to support communication of different criticality levels. TTEthernet coordinates transmission of all three traffic classes over the same physical connections. In this way, all traffic classes may coexist within one network.

Time-triggered (TT) messages are particularly suited for communication in hard real-time control systems. Still, considerable effort is required to design the communication schedule upfront, and changes to the network configuration necessitate changes to the schedule. The rate-constrained (RC) traffic class instead uses an event-driven paradigm, providing increased flexibility for applications with less stringent latency and determinism requirements. Rate-constrained messaging is analogous to the communication method specified by the ARINC 664-p7 standard and seen in a wide range of aerospace applications. It uses the same virtual link concept for message delivery as the time-triggered class, as well as the same Ethernet frame structure. 
The frame payload size and rate of transmission are limited to predetermined maximums for each virtual link, and message delivery is guaranteed. ${ }^{8,22}$ The configuration of switch buffers can be adjusted to accommodate the known worst-case traffic pattern and thus buffer overflows can be eliminated. ${ }^{20}$ Still the event-triggered nature of rate-constrained traffic requires that switches arbitrate between conflicting frames. As a result, transmission latency is more variable than that of the time-triggered class. The best-effort (BE) traffic class is also event-driven, and behaves akin to the messaging approach taken by classical Ethernet. No guarantees are provided regarding transmission latency or successful message delivery. ${ }^{22}$ Best-effort messaging is thus only appropriate for communication of nonessential data.

All three traffic classes can be integrated together within the same network. Time-triggered communication is the highest priority. Its transmission cannot be hindered by the presence of any event-driven traffic ( $\mathrm{RC}$ or $\mathrm{BE})$. In the case that an end system chooses not to send a time-triggered message during its allocated slot, the switch reclaims the bandwidth for use by event-based traffic. ${ }^{22}$ Event-driven traffic may use any bandwidth not taken by time-triggered messages. Out of the event-driven classes, rate-constrained traffic is always prioritized over best-effort traffic. Any best-effort messages are therefore restricted to the leftover bandwidth not used by the higher priority classes.

Figure 4 shows the integration of multiple traffic classes within a TTEthernet network. Notice that TT messages are forwarded as scheduled without delay, while event-driven traffic is transmitted as priority allows.
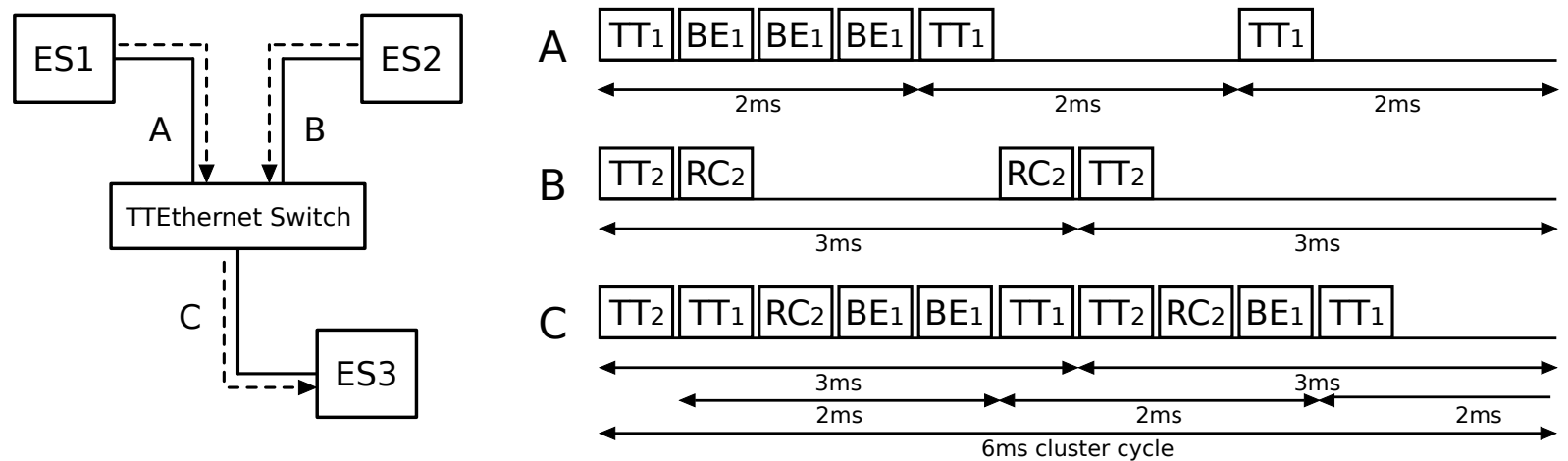

Figure 4. Integrated dataflow in a sample TTEthernet network.

Efforts must be taken to ensure determinism of high priority messages in a network transporting frames of differing criticality levels over the same physical layer. Conflicts between traffic classes could arise if a lower priority message is in the middle of transmission when a higher priority message becomes ready to send on the same physical port. TTEthernet provides two methods by which to address this issue. The first, called timely block, prevents RC or BE messages from being sent during time slots allocated for timetriggered messages until: 1) transmission of the TT message completes or 2) the switch can verify that no TT message will be sent in the time slot. The use of timely block is limited to time-triggered traffic, as it requires that the transmitting device have advanced knowledge of when the higher priority-message may be sent. The second method, called shuffling, can prevent conflicts between any of the three traffic classes. In shuffling, the higher priority message is temporarily stored in a queue until the lower priority message is finished transmitting. ${ }^{22,23}$ The timely-block method is generally preferred when sending time-triggered messages, as the variable delay introduced by the shuffling method is not acceptable in most time-critical applications.

\section{VII.C. Synchronization Process}

The SAE AS6802 standard describes a time-triggered Ethernet synchronization protocol designed to establish and maintain a common time base between distributed network components (e.g. switches and end systems). A group of devices whose local clocks are synchronized in order to enable time-triggered communication is referred to as a synchronization domain. It is possible to design a TTEthernet network containing multiple independent synchronization domains. Communication between these clusters, however, is possible only through the use of event-driven traffic classes ( $\mathrm{RC}$ or $\mathrm{BE}$ ). 
Each component (e.g. switch, end system) within a synchronization domain is assigned one of three roles corresponding to its purpose during synchronization: Compression Master (CM), Synchronization Master (SM), or Synchronization Client (SC). The information necessary to establish and maintain synchronization between TTEthernet devices within a domain is transmitted in protocol control frames (PCF). Each synchronization role utilizes these PCFs in different ways as part of the overall synchronization process. Switches are typically configured as CMs, while end systems generally act as SMs. ${ }^{24}$

Synchronization is first established within the network by means of a fault-tolerant handshake between the SMs and CMs. The SCs act simply to relay PCFs to their intended destination. The SMs dispatch specialized coldstart frames to the CMs. The CMs then relay the messages back to the SMs, prompting the transmission of a coldstart acknowledgement frame indicating the start of synchronized operation. Once a global time base has been established, it must be adjusted at regular intervals, called integration cycles, to account for clock drift within the network devices. ${ }^{23,24}$

This process begins with all SMs dispatching PCFs to the CMs at the same local time. As the local times on each SM will have slightly diverged since the last resynchronization, the PCFs will actually be sent and subsequently received by the $\mathrm{CMs}$ at different points in time. The CMs then use this variation in $\mathrm{PCF}$ reception time to calculate a new average global time. ${ }^{22,24}$ This global time is relayed back to both the SMs and SCs in another PCF, resynchronizing each devices local clock. Furthermore, TTEthernet provides means to detect and correct the loss of synchronization between devices (e.g. clique detection). ${ }^{22}$

Figure 5 shows the transmission of PCFs within an example TTEthernet synchronization domain. All three end systems are configured as synchronization masters. One

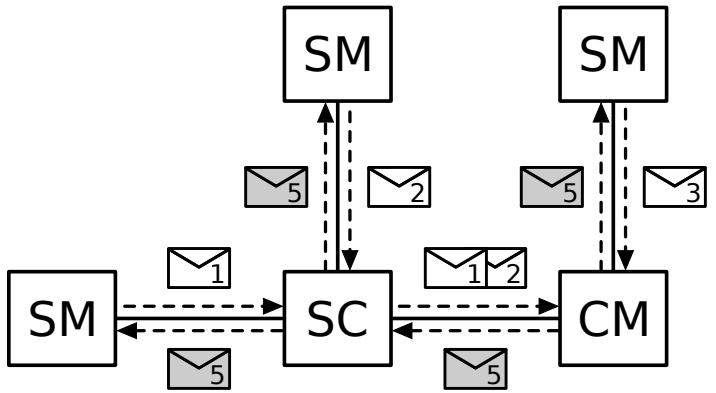

Figure 5. Flow of protocol control frames in simple TTEthernet network. switch acts as a synchronization client, while another acts as a compression master.

The TTEthernet protocol is designed to tolerate a variety of end system and switch failures (e.g. arbitrary or inconsistent omission) without a degradation of essential services such as synchronization. In the most extreme case, TTEthernet networks can be configured to tolerate the failure of any two network devices (dual-fault tolerant). ${ }^{24}$

\section{TTEthernet Flight Computer Failover}

This section explores the application of TTEthernet technology in two separate demonstrations of realtime failover between redundant flight computers running CFS in a simulated spaceflight mission. The first was an Asteroid Redirect Mission (ARM), in which flight computers controlled the Orion spacecraft (with ESA's ATV-derived service module) as it traveled over one thousand meters to dock with a robotic asteroid retrieval vehicle. Within this scenario, the flight computers were required to communicate using traditional Ethernet with two spacecraft systems external to the flight control loop - a crew display and Environmental Control and Life Support System (ECLSS) simulator. The second mission, an Orion Ascent Abort 2 (AA2) flight test, simulated activation of the Launch Abort System (LAS) at the point where the Space Launch System (SLS) undergoes the highest degree of aerodynamic stress (max Q). The LAS carried the Orion crew module several thousand feet away from the rocket before reorienting the vehicle and detaching from the capsule. Unlike the ARM mission scenario, the AA2 test focused entirely on communication between the flight computers and simulation. Several significant improvements to the TTEthernet-based network architecture used in the ARM mission were made to meet the more pressing timing and data requirements of the AA2 scenario. Software-based Internet Protocol version 4 (IPv4) and User Datagram Protocol (UDP) layers were developed to accommodate the larger message payload sizes. Furthermore, flight computer synchronization was implemented to ensure that all redundant machines processed data concurrently, and to enable failover between computers while guaranteeing no skipped or duplicated commands.

Next, prior efforts to demonstrate flight computer failover are presented and motivation for the implementation of a TTEthernet-based failover architecture are discussed. The following subsections then describe each key aspect of the ARM and AA2 demonstrations: 1) the structure of the flight software and flight con- 
trol loop, 2) the integration of TTEthernet messaging into CFS, 3) the application of different failover and synchronization methods, 4) the integration of TTEthernet with traditional IP-based Ethernet networks, and 5) the development of supporting software applications. Lastly, the setup of each failover demonstration is described as shown in the A\&S project's September FY14 (ARM) and May FY15 (AA2) Integrated Tests at NASA JSC's IPAS facility.

\section{VIII.A. Background and Motivation}

Implementing a fault-tolerant avionic system often necessitates the use of multiple distributed computing platforms capable of performing identical tasks. The system is designed such that vehicle health is maintained despite the loss of one or more of these individual computers. This redundancy is especially important within architectures designed by IMA principles, as each computer system may control multiple critical vehicle functions. Failover is one fundamental method of failure recovery in such distributed aerospace systems. Systems designed to carry out failover scenarios use the concept of primary and backup agents. When the primary agent experiences a critical fault, its responsibilities are taken over by the backup through a failover procedure. $^{25}$ The ideal result of this failover process is the return of standard vehicle functionality without degraded performance. Failover among identical platforms is particularly suited to mitigate the effects of both random and end-of-life hardware failures. ${ }^{26}$ The Space Shuttle was the first major aerospace project to implement automated failure detection and recovery. It employed a redundant set of four flight computers, each coordinating with specific sensors and effectors over a subset of vehicle data buses (depending on the program loaded). A separate backup flight computer was capable of communicating on all data buses in case of failures during critical mission phases (e.g. docking, reentry). ${ }^{27}$

Though failover methods are designed to allow backup flight computers to assume control of vehicle systems, physical sensors and actuators are not always available in simulated mission environments. The Trick Simulation Environment, locally developed at JSC, is used to model these absent systems (e.g. inertial measurement units) within the IPAS testbed. Trick is also used to model the vehicle's trajectory through space and the dynamics of any other bodies of interest (e.g. service module, asteroids). The flight computer hardware can therefore interface directly with Trick in order to conduct simulated missions. Trick simulation software has been used to support many NASA programs over the last several decades, including the Space Shuttle, ISS, and Orion. ${ }^{28}$ Furthermore, the AA2 flight test simulation achieves high fidelity through utilization of the Advanced NASA Technology Architecture for Exploration Studies (ANTARES) simulation, the official NASA Orion spacecraft assessment tool used by JSC's Guidance, Navigation, and Control (GNC) branch. ANTARES consists of several separately compiled models, packages, and libraries executed by the Trick simulation environment. ${ }^{29}$

Past efforts by the A\&S project to demonstrate failover between flight computers utilized the existing IP-based Ethernet backbone. Because other vehicle systems were designed only to communicate with one flight computer IP address at a time, a load balancer was needed to act as a virtual flight computer IP and direct messages to/from both machines. This solution was demonstrated across multiple operating systems (e.g. Linux 2.6, VxWorks 6.9) and hardware platforms (e.g. non-flight-like, Maxwell SCS-750). ${ }^{6}$ Still, it suffered the typical problems associated with using classical Ethernet for time-critical communication and provided minimal fault-tolerance by introducing a single point of failure. While IP/Ethernet systems can achieve greater fault-tolerance through a variety of means (e.g. redundant load balancers, Virtual Router Redundancy Protocol (VRRP)), these methods ultimately rely on devices to monitor each other through the network, and take over as necessary. Because this monitoring is dependent on the rules of traditional best-effort Ethernet, there are no guarantees regarding successful message delivery or travel time. Such systems are therefore inappropriate for use in critical applications.

TTEthernet was selected for subsequent iterations of the failover architecture as part of the A\&S project's ongoing efforts to introduce deterministic Ethernet technology to future spacecraft designs. Besides the general benefits of time-triggered Ethernet described earlier, some aspects of the technology make it particularly well-suited for this application. First, TTEthernet's use of virtual link based message forwarding removes the need for a separate load balancer, as simple adjustments to the communication schedule ensure identical messages can be dispatched to several recipients simultaneously. These time-triggered channels also allow failover to occur more seamlessly. Both the primary and backup computers are guaranteed to receive identical data from the simulation at specific time intervals. This means that at the point in time that the primary computer is lost, the backup flight computer can begin reacting to the next set of fresh simulation data with no interruption. Additionally, the static nature of VL message paths ensures that the communication loop 
between the flight computers and the simulation remain closed, while keeping the flight computers accessible to other systems on the Ethernet backbone. Redundant TTEthernet switches can also be used to increase fault-tolerance. These switches can be hot-swapped with no negative impact on the running simulation. Finally, the periodic nature of time-triggered communication integrates well with CFS's own schedule driven architecture.

\section{VIII.B. CFS Structure and Control Loop Setup}

The implementation of TTEthernet-based failover requires integration of the TTEthernet library with the flight software running on each flight computer. The flight software is a derivative of the CFS product line maintained by Johnson Space Center's Spacecraft Software Engineering Branch and used extensively in the IPAS environment. The integration of TTEthernet with CFS is important not just for this failover application, but also so it may be leveraged in future spaceflight projects.

CFS provides a mission independent environment offering core services designed to insulate developers from underlying hardware and software dependencies. The long-term goal of CFS's integrated core flight executive (cFE) is to facilitate the growth of a reusable bank of flight software applications. ${ }^{30}$ Because the requirements for command and data handling $(\mathrm{C} \& \mathrm{DH})$ flight software are similar from project to project, the cost and turnaround time of future software development can be lowered through code reuse. ${ }^{31}$ Johnson Space Center is particularly interested in extending CFS's capabilities for use in manned spaceflight applications. The integration of TTEthernet facilitates the development of additional CFS applications that may communicate in safety-critical or mixed-criticality networks generally required by such projects.

The CFS product lines follows a layered architecture described in Ref. 32. Each layer masks the specifics of its underlying operation and can be changed without impacting the other layers. ${ }^{31}$ Among the bottom layers is a hardware abstraction layer (HAL), offering a common library designed to provide plug and play capability for qualified processors and other devices. The board support package (BSP) layer boots this HAL, as well as the CFS and chosen operating system. Above this, the operating system abstraction layer (OSAL) provides a library interface enabling CFS compatibility with different operating systems. One layer higher is the $\mathrm{cFE}$ itself, implementing six fundamental services required by flight software applications: Executive Service (ES), Software Bus Service (SB), Event Service (EVS), Table Service (TBL), File Service (FS), and Time Service (TIME). Lastly, the top layer houses all flight software applications. These include both pre-validated mission-independent modules and other applications tailored to a specific project. ${ }^{32}$

The CFS product line houses all essential flight software, including the $\mathrm{cFE}$ and all lower layers. Projectspecific applications are instead located in the CFS workspace pertaining to an individual mission. These applications communicate through the software bus (abstracted from sockets) implemented by the underlying cFE. This bus follows a loosely-coupled publish-subscribe model, providing a structured architecture in which each application requires no knowledge of the underlying communication method (e.g. protocol, destination). ${ }^{33}$ The applications are scheduled to run periodically according to a global scheduling table. The CFS scheduler sends "wake up" messages to each application at preconfigured time intervals. Upon receiving this message, an application executes exactly once. Typical schedules run at frequencies ranging from $100 \mathrm{~Hz}$ to $500 \mathrm{~Hz}$. An adjustable script file controls which applications are loaded at start up and subsequently run within the flight software.

Additional components are needed to actually conduct a simulated mission. The simulated vehicle's GNC flight software is generated using a process similar to that used for the actual Orion spacecraft. The MathWorks MATLAB and Simulink tools are used to auto-generate $\mathrm{C}++$ source code from GNC algorithms. This code is compiled into a library and accessed from a CFS module housed in the CFS workspace corresponding to the given project. The Multi-Purpose Crew Vehicle (MPCV) GNC workspace used in the TTEthernet ARM failover test incorporated the genuine Orion Absolute Navigation (AbsNav) code for Exploration Mission 1 (EM-1). ${ }^{28}$ The code used for the TTEthernet AA2 failover demonstration added several improvements, including service module abort functionality, stochastic and optical navigation, and propellant balancing in the crew module's thruster logic.

Moreover, there are three ways by which the flight computers may communicate over the data network: 1) using the software bus (in the case of distributed flight computers), 2) via standard telemetry input/output streams, or 3) using purpose built interface applications (housed in the workspace) to send and receive messages directly. By default, a flight computer and Trick simulation communicate using the third of these options. This control loop employs dedicated "sim-to-fsw" and "fsw-to-sim" CFS applications to relay IP messages with UDP or TCP transport layers depending on the requirements of the mission. 


\section{VIII.C. CFS TTEthernet Integration}

In order to replace the closed loop's IP-based communication method with time-triggered messaging, TTEthernet end systems realized on Altera FPGAs were installed in the simulation machine and each redundant flight computer. Furthermore, a shared CFS library was developed to provide compatibility between CFS and the new TTEthernet network adapters. This "hardware servicing" library interfaced applications with the TTEthernet vendor library, which in turn interacted directly with the TTEthernet driver. The driver was implemented as a loadable kernel module. The library was built along with the various flight software applications and loaded according to the same CFS startup script. It allowed any CFS application within the workspace to access core TTEthernet capabilities, including the ability to send and receive mixed-criticality messages. This library also performed setup or cleanup procedures automatically when CFS was initiated or shutdown.

An extended vendor library was created to meet the needs of the AA2 flight test, including: 1) message payloads sizes up to 20,000 bytes and 2) throughput rates up to 100Mbit/s per Ethernet link. To satisfy the first requirement, the extended library included $80+$ additional functions implementing a complete softwarelevel network stack featuring IPv4 and UDP protocol layers. The extended library's API replaced the default vendor-provided TTEthernet API, allowing applications to send or receive messages encapsulated as UDP datagrams and $\mathrm{IPv} 4$ packets as TT, RC, or BE traffic. ${ }^{\mathrm{b}}$ When all protocol layers are utilized, message payloads up to 65,507 bytes can be transmitted over the network. This value is determined by: $(65,535$ theoretical UDP length limit $)-(8$ byte UDP header $)-(20$ byte IPv4 header $)=65,507$. The IPv4 and UDP layers were built according to Internet Engineering Task Force (IETF) publications RFC 791 and RFC 768 respectively. ${ }^{34,35}$

The second requirement was addressed by the direct memory access (DMA) capabilities of the TTEthernet end systems. Communication through the TTEthernet library is possible using either programmed input/output (PIO) or direct memory access (DMA). All incoming and outgoing transfers using the PIO mechanism must travel through the processor, limiting throughput to roughly $10 \mathrm{Mbit} / \mathrm{s}-30 \mathrm{Mbit} / \mathrm{s}$. DMA, however, enables full gigabit transmission speed by allowing the TTEthernet controller to access system memory independently of the host processor. The extended version of the TTEthernet library provides a higher level of abstraction around use of TTEthernet's DMA transfer mechanism, helping developers avoid common pitfalls when using the DMA interface to send/receive messages.

Five automated tests were conducted to characterize the performance of $\mathrm{RC}$ and $\mathrm{BE}$ read/write operations within the extended TTEthernet library. Time-triggered traffic was omitted because: 1) the overhead produced by the network layers for TT traffic is identical to RC traffic, and 2) the transmission rate of TT traffic is governed by the TTEthernet network schedule used.

Messages were transmitted between two identical HP Z400 desktop computers (3.2GHz Xeon QuadCore W3565, 4GB RAM, CentOS 6.5 64-bit) through a 1Gbit/s 12-port TTEthernet development switch. Each test was broken into 60 distinct 1 minute trials, during which one computer continuously transmitted messages of a particular protocol and traffic class to the receiving computer at a specific transfer rate. The message payload sizes used were selected to represent common data lengths for which each traffic class might be utilized.

The transfer rate was increased linearly from $0 \mathrm{Mbit} / \mathrm{s}$ to $1 \mathrm{Gbit} / \mathrm{s}$ and verified using a Fluke Optiview XG Network Analysis Tablet. For the RC traffic tests, a band allocation gap (BAG), the minimum average time between the transmission of consecutive frames, was set to $1 \mu \mathrm{s}$ in the TTEthernet schedule. Though $1 \mathrm{~ms}$ is the smallest BAG permitted while maintaining ARINC 664-p7 compatibility, such a large delay would severely limit the potential transfer rate of $\mathrm{RC}$ traffic for the tests. A $1 \mu \mathrm{s}$ BAG delays messages the smallest amount possible while still performing traffic shaping, that is, keeping the traffic rate-constrained (TTEthernet supports arbitrary BAGs with a granularity of $1 \mu \mathrm{s}$ ). ${ }^{36}$ The extended TTEthernet library was configured with a maximum IP fragment payload size of 1480 bytes. Together the minimum BAG and maximum fragment payload size minimize the time required to transmit all fragments of a given payload, therefore maximizing the net communication rate for both $\mathrm{RC}$ and $\mathrm{BE}$ traffic.

After each trial finished, both the number of messages sent and the number of messages successfully received were recorded. Once all 60 trials were completed, the percentage of successfully received messages was calculated for each transfer rate. The percentage of received messages was then interpolated using

\footnotetext{
${ }^{\mathrm{b}}$ The Phoenix TTEthernet Intellectual Property (IP) used for this project operates only at the Media Access Control (MAC) layer (OSI Layer 2). The newer Pegasus TTEthernet IP implements network and transport protocol layers for the TT, RC, and $\mathrm{BE}$ traffic classes.
} 
Piecewise Cubic Hermite Interpolating Polynomial (PCHIP) interpolation to produce a continuous plot. PCHIP interpolation was chosen to avoid overshoots/undershoots in the resulting graph. Figure 6 displays the graphs produced as a result of all five throughput tests.

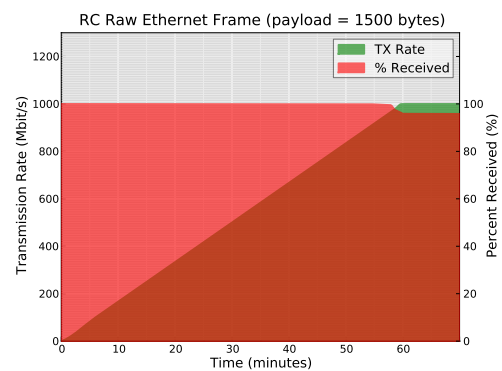

(a)

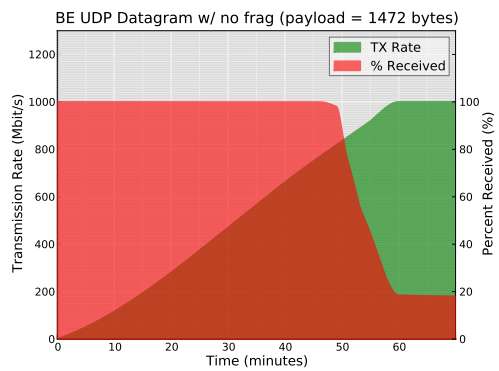

(d)

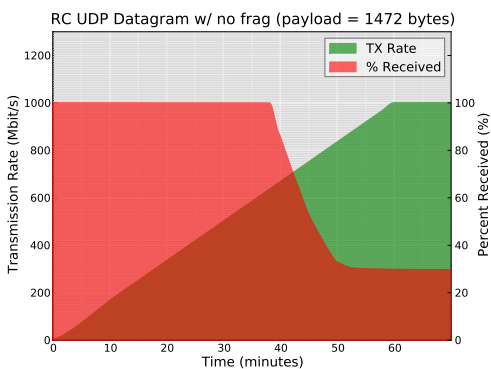

(b)

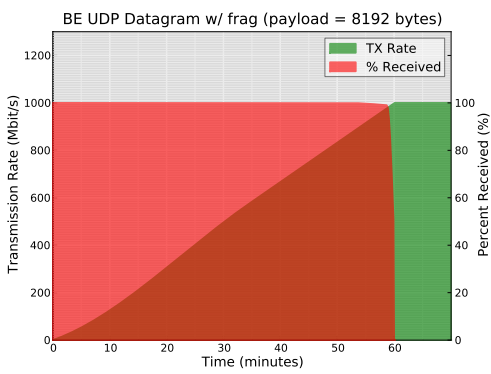

(e)

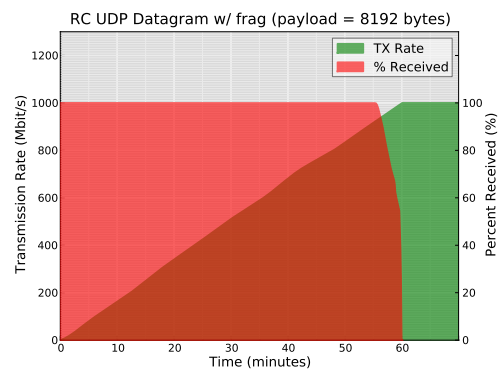

(c)

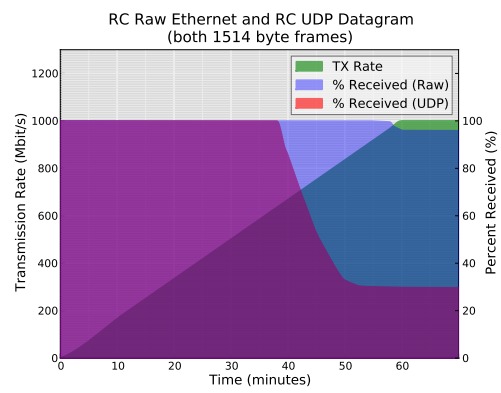

(f)

Figure 6. Throughput analysis for RC and BE traffic in the TTEthernet library extension.

These plots can be used to determine the maximum effective throughput for each test - the highest possible transmission rate at which no messages are dropped by the receiver. This value identifies a realistic upper throughput limit permitted by the extended TTEthernet library for a particular protocol, traffic class, and payload size combination on the specific HP Z400 platform used for these tests. Because the IPv4 and UDP layers were implemented in software, however, performance can vary significantly depending on the hardware and operating system on which the extended TTEthernet library is utilized. Still, the high performance seen in the above results demonstrates the merit of implementing an application-level network stack on top of TTEthernet's data-link layer.

For both the simulated ARM mission and AA2 flight test, the "sim-to-fsw" and "fsw-to-sim" CFS applications were rewritten to send and receive time-triggered messages using functions in either the stock (for ARM) or extended (for AA2) TTEthernet library. The Trick simulation was also modified to communicate using time-triggered messages. In the simulated ARM mission, the "fsw-to-sim" application transmitted vehicle control commands to the Trick simulation at a frequency of $5 \mathrm{~Hz}$, while the "sim-to-fsw" application read situational data from the simulation at a frequency of $10 \mathrm{~Hz}$. The AA2 test required tighter coupling between the flight computers and simulation, and thus ran both the "fsw-to-sim" and "sim-to-fsw" applications at a much faster $40 \mathrm{~Hz}$ rate.

For the AA2 mission, the CFS scheduler application was rewritten to leverage the global synchronized TTEthernet clock maintained between the flight computers, simulation computer, and network switches. CFS applications are executed at specific instances according to a predefined schedule table consisting of a fixed number of slots (e.g. 100, 200). Each slot references a platform dependent number of message ID entries - each associated with a distinct application to be executed when that slot is reached. Interrupts generated by CFS's Platform Support Package (PSP) are used to move between slots of the schedule at a constant rate. Minor frames are defined as the individual windows created between these interrupts, in which the active applications may perform their work. The aggregate of all minor frame windows comprises the major frame, or the period of the entire CFS schedule table (generally 1 second). When a given slot is reached, the scheduler dispatches "wake up" software bus messages to the message IDs referenced within each slot entry, causing each corresponding application to execute.

One significant limitation of the default CFS scheduler was the need to correct for the relatively poor 
accuracy of the timing base driving minor frame transitions. ${ }^{\mathrm{c}}$ To meet the tight timing requirements of a highly packed schedule consisting of 200 slots or more, the scheduler would sometimes process multiple slots at once in an effort to remedy the inaccuracy of the underlying clock signals. This behavior resulted in irregular minor frame lengths and inconsistent times at which applications execute. Furthermore, it hindered the ability to synchronize multiple redundant flight computers without the introduction of significant additional complexity. The global synchronized time base maintained by the TTEthernet services provides a much higher degree of clock accuracy and can thus be leveraged to drive the CFS schedule and overcome this problem. The new CFS scheduler application used for the AA2 flight test drove slot transitions according to the repetition of cluster cycles within the TTEthernet network. The cluster cycle period is defined as the least common multiple of all time-triggered communication periods. The AA2 CFS schedule was developed with 200 slots and a major frame period of 1 second. Progression between slots therefore had to occur at regular $5 \mathrm{~ms}$ intervals. The TTEthernet network schedule was designed with a cluster period of $250 \mu \mathrm{s}$. Within the CFS scheduler, interrupts from the TTEthernet controller were generated at the start of each new cluster cycle. A callback function was used to process the next slot in the CFS schedule every 20 interrupts $(250 \mu \mathrm{s} \times 20=5 \mathrm{~ms})$. Functions to calculate the number of interrupts comprising one minor frame using the cluster period and total CFS slot count were added to the extended TTEthernet library for use in future projects.

\section{VIII.D. Flight Computer Failover and Synchronization Methods}

Failover mechanisms between redundant computers are generally classified as either blocking or non-blocking protocols. In both cases, the state of the backup machine must mirror the state of the primary. This is usually accomplished via transmission of status information from the primary to the backup whenever the primary machine must service a request. In a blocking protocol, the backup machine processes this status update and relays an acknowledgment message back to the primary. Only upon receipt of this acknowledgment may the primary machine respond to the original service request. Alternatively, non-blocking protocols do not require the primary machine to wait for acknowledgment from the backup before servicing requests. This lack of idle wait time typically results in higher performance, but can lead to loss of synchronization (between computers) if messages are lost. ${ }^{25}$ With a properly configured TTEthernet network, however, delivery of this critical data is guaranteed. For these reasons, all failover methods discussed in this section are non-blocking.

For the ARM mission, two methods were developed to enable failover between two redundant flight computers over a TTEthernet network - one based on periodic heartbeat messages, and the second using coupled data pairings. The failure of a particular machine was realized by ceasing execution of its flight software.

In the first failover method, the primary computer sends time-triggered heartbeat messages to the backup machine to indicate that it is still functioning. If the backup stops receiving heartbeats, it assumes that the primary machine has failed and takes control of the vehicle. Because both flight computers receive the same data from the simulation simultaneously, the exchange of no other state information is necessary. Each flight computer sends or receives heartbeats using purpose built CFS applications scheduled to execute at a $10 \mathrm{~Hz}$ rate. This schedule is designed such that each heartbeat transmission is paired with its subsequent reception on the backup machine. Though a simple solution, the heartbeat-based failover method suffers from a susceptibility to unpredictable timing variations with the flight control loop - both regarding the occurrence of a failure and the execution of the flight software on each machine. When the primary computer fails, an unpredictable time delay occurs before the backup recovers vehicle operation. Because the heartbeat listener is realized within a CFS application, the backup machine can only periodically check the status of the primary computer. This introduces obvious timing unpredictability, as the delay experienced during failover is directly related the failure's proximity in time to the next scheduled read. This variability is further impacted by a lack of synchronization between the CFS schedules running on each flight computer. Even though each computer processes the same simulation data, their exact placement within the CFS control loop may be staggered an unpredictable amount. No guarantee exists that the backup's "read heartbeat" application will execute immediately after the primary's "send heartbeat" application.

Consider a case where heartbeats are both dispatched from the primary computer and read from the backup at a typical $100 \mathrm{~ms}$ interval in a 200 slot schedule with a 1 second major frame. For simplicity, assume that the transmission time of the TT messages is instantaneous. The CFS schedules loaded on each

\footnotetext{
${ }^{\mathrm{c}}$ Several more deterministic CFS schedulers have been developed at Johnson Space Center for use with NASA flight projects (e.g. Project Morpheus).
} 
computer exactly match, with the same slots on each machine used to either send or receive heartbeats (made possible due to the instantaneous delivery). Also say the primary machine fails directly after a heartbeat is sent, and that the backup seizes control of the vehicle immediately after failure is detected. If the flight software schedules are offset such that the read takes place $99 \mathrm{~ms}$ after the heartbeat is transmitted, $199 \mathrm{~ms}$ will pass after failure occurred before the backup takes over as the primary machine. Moreover, the backup computer will process 19 CFS schedule slots before detecting the failure. The behavior of the applications executed in these slots, however, could change significantly depending on the role of the host computer (e.g. only the primary computer sends effector commands to the simulation). Now imagine that the schedules are staggered such that the read takes place $1 \mathrm{~ms}$ after the heartbeat is sent. The backup won't take over for $101 \mathrm{~ms}$ after the primary computer went down (again processing 19 slots). On the other hand, if the primary computer instead failed directly before it would have transmitted a heartbeat, the backup could detect the failure only $1 \mathrm{~ms}$ later. This unpredictable behavior can be mitigated by increasing the rate at which both the sender and receiver heartbeat applications are run. However, this introduces additional system complexity and limits the number of time slots available for other flight software applications.

The second failover method used for the ARM mission better leverages TTEthernet's capabilities to increase performance and eliminate the need for a periodic heartbeat. Both flight computers still receive data from the simulation simultaneously, but are no longer divided into static primary and backup roles. Because no distinction exists between them, each machine processes information and sends effector commands back to the simulation as if it is the only computer flying the vehicle. The use of parallel data paths from multiple flight computers is similar to the approach taken by the Orion spacecraft, in which both vehicle management computers (VMCs) simultaneously transmit commands to PDUs controlling redundant effectors. Both messages are sent back such that the simulation always receives them in paired sets. The receipt of both commands composes one single command cycle. In each cycle, only one computer is designated as primary. The simulation processes commands only from each cycle's primary machine, discarding data sent by the backup. If no command from the primary computer is included in the received data set, the backup computer takes the primary designation, its command is processed, and the mission continues uninterrupted.

Still, a lack of synchronization between computers leads to unpredictable delays and the potential for either repeated or skipped CFS schedule slots. After a failover occurs, the new primary flight computer could begin running applications at a slot the previous primary machine had already processed. Similarly, the new primary machine may begin processing at a slot the previous had not yet reached. Both of these cases could have severe repercussions, including duplicated jet firings and incorrect sensor readings. Furthermore, the method's use of the simulation machine to arbitrate between commands sent from each flight computer can often not be realized on actual flight hardware (e.g. Ethernet I/O modules driving actuators may not accommodate redundant commanding).

For the AA2 flight test, a priority-based master/slave framework was developed to combine: 1) redundant flight computer synchronization and 2) a traditional heartbeat-based failover mechanism. Like in the failover methods described previously, all flight computers receive data from the simulation simultaneously. Also, as in the heartbeat-based failover method used for the ARM mission, only one flight computer at a time is capable of actually driving the simulation and controlling the vehicle. Unlike those described earlier, however, the failover method developed for the AA2 flight test was built to potentially utilize a larger set of redundant computers (e.g. three, four, or more). Actual spacecraft control systems generally require four or five redundant flight processors to achieve the desired level of fault tolerance.

All computers in the redundant set run the same Core Flight Software load, with the exception of a single configuration file that specifies: 1) the machine's communication interfaces (i.e. the virtual links) and 2 ) the computer's priority within the set. The priority assigned to each machine ranges from 1 (highest priority) to $\mathrm{N}$ (lowest priority), where $\mathrm{N}$ designates the total number of flight computers. The behavior of each flight computer is determined by its priority. All computers with priorities $2-\mathrm{N}$ act as slaves on the network, waiting to synchronize their own flight software execution to that of the highest priority machine. By contrast, the highest priority computer acts as the master, drawing from the TTEthernet time base to drive the execution of its flight software schedule and control the simulated vehicle. Each time its CFS scheduler processes a given slot (i.e. executes the applications the slot references), it commands all lower priority machines to process that same slot. Unlike in previous methods, in which each flight computer ran according to its own internal timers, the execution of the CFS schedule on one machine cannot become staggered from the rest of the set. Thus the flight software on each computer executes simultaneously.

The failure of a slave computer does not negatively impact the mission, since only the master computer 
dispatches effector commands processed by the simulation. The master computer is assumed dead by a slave if no command to process a new slot has been received, and the elapsed time since the last slot was processed surpasses a user-configurable timeout value. When running CFS on a non-deterministic Linux OS, a conservative timeout limit is twice the length of the CFS schedule's minor frame. For the AA2 flight test, in which all computers employed a 200 slot CFS schedule, a timeout of $10 \mathrm{~ms}$ was used $(2 \times 5 \mathrm{~ms}$ minor frame $=10 \mathrm{~ms}$ ). The smallest timeout needed to avoid false positives (i.e. incorrectly asserting the master has failed) can shrink as the number of slots in the CFS schedule increases. For example, a flight computer running a 500 slot CFS schedule could use a smaller $4 \mathrm{~ms}$ timeout $(2 \times 2 \mathrm{~ms}$ minor frame $=4 \mathrm{~ms})$.

Each slave computer maintains a list of failed masters and references its own priority level to determine how to react if the master computer is lost. If the master fails, the next highest priority machine assumes the role of the master - controlling the vehicle and driving the flight software execution of all remaining computers. Unlike in the previous failover methods discussed, no slots are skipped or repeated as a result of this failover process. All lower priority slave computers recognize that a higher priority computer in the set is still alive and listen for commands from the new master. As a result, all remaining computers in the set maintain synchronization with one another.

\section{VIII.E. Integration with Traditional IP-based Networks}

One challenge faced when integrating the TTEthernet-based flight computers into the IPAS environment is the need to maintain compatibility with all systems already configured for use with the existing Ethernet backbone. Besides the Trick simulation, the flight computers must communicate with several non-flight stand-ins for actual Orion systems. These include an ECLSS simulator, a propulsion system featuring 16 cold gas jets, and power generation and distribution systems. Communication between these systems and the flight computers occurs via the platforms' available IPS network stacks, which are accessible through standard socket programming techniques. Ideally, the TTEthernet controller would be used for communication of these classical Ethernet messages.

Communication within traditional Ethernet systems, however, generally requires higher network (e.g. IP) and transport (e.g. TCP, UDP) layers on top of Ethernet's data link layer. These layers provide essential services, such as addressing, routing, segmentation, and acknowledgement. TTEthernet devices can maintain compatibility with systems employing the Internet Protocol Suite by implementing the necessary higher layer protocols over the best-effort traffic class.

The TTEthernet NetDev driver was used to meet this need for the simulated ARM mission, allowing a TTEthernet controller to act as a standard Ethernet network interface in Linux systems. It leverages the Linux kernel's Berkeley Software Distribution (BSD) IPS implementation to communicate using IP packets encapsulated in best-effort frames. The NetDev driver is implemented as a standard Linux kernel module and is loaded in addition to the TTEthernet driver. Parallel transmission of both critical traffic (via the TTEthernet API) and classical Ethernet traffic (via NetDev) is possible as long as no shared resources are present (e.g. input/output buffers). Unfortunately, throughput via the NetDev driver is limited due to its use of the PIO transfer mechanism (10Mbit/s - 30Mbit/s). For the ARM mission, the bandwidth necessary for communication with the various IPAS subsystems fell below this threshold, and use of the NetDev driver was therefore acceptable. The NetDev driver simplified the integration of TTEthernet into the IPAS testbed, as pre-existing IP-based applications (both on the flight computers and in the simulated vehicle subsystems) not involved in the flight control loop required no modifications.

The vendor library extension created for the AA2 flight test mostly eliminates the need for the NetDev driver, implementing application level IPv4 and UDP protocol layers, as well as providing a high level interface to TTEthernet's DMA transfer capabilities. The extended library therefore overcomes NetDev's speed limitation and allows TTEthernet devices to communicate natively with IP-based systems. No separate drivers or libraries are necessary. Still, in order to simplify its design, the library does not include all protocols found in a full implementation of the Internet Protocol Suite. Most notably, the following protocols are not present: 1) Address Resolution Protocol (ARP) and 2) Internet Control Message Protocol (ICMP). Because the library extension does not utilize multithreading, the use of these protocols would introduce significant computational overhead and limit overall network throughput. Instead, IP address to MAC address mapping is accomplished via a statically configured text file loaded at runtime. The error detection and reporting services traditionally provided by ICMP are entirely absent. Though the use of best-effort traffic was not required as part of the AA2 flight test, the library can be used to enable communication with traditional Ethernet-based vehicle systems in future simulated mission scenarios. 


\section{VIII.F. Supporting Software}

Several different software applications were developed to aid in the configuration of TTEthernet networks and in the visualization of their operation.

The first program, a TTEthernet network card status monitor written in C, was created to provide real-time feedback regarding the condition of any installed TTEthernet controllers. Motivation for building the application stemmed from the need to quickly identify the health and operational state of end systems involved in closed loop time-triggered communication. The program is capable of accessing a variety of information about the installed network adapters through the TTEthernet API. This data includes the cards' schedule configuration information, synchronization status, and error state. A complementary desktop graphical user interface (GUI) was developed with the GTK+ toolkit. This allowed the program to maintain compatibility with other platforms (e.g. UNIX, OSX) and to fit naturally within Linux OS's using the GNOME desktop environment (e.g. RHEL). The GUI displays a bank of icons representing the network cards' status in different broad categories (e.g. Synchronized, Error). The status monitor program acts as a passive listener on the host device, updating this display in real time as other applications communicate over the TTEthernet interface. A user is thus able to visualize the progression of an end system as it joins the network, synchronizes with other devices, and begins transmitting messages. Errors, like lapses in synchronization, are also shown. All this information can be timestamped according to TTEthernet's synchronized global clock and logged to a file for later viewing. When combined, the status logs produced by each end system provide a helpful resource in determining the cause of any faults or unexpected network behavior.

A second application (again written in $\mathrm{C}$ and using GTK+) was developed to let a user change a local end system's schedule configuration from a straightforward graphical interface. This separates the management of a device's communication schedule from the software actually used to send and receive TTEthernet messages. Within the context of a redundant flight computer setup, it allows both computers to run identical flight software loads while still communicating according to different TTEthernet schedules. If the schedules change, no update or recompilation of the flight software is needed. The program displays all TTEthernet controllers installed in a local system. For each card, it shows both the number of available communication channels and status information similar to that provided by the status monitor application. Through the point and click interface, a user can select a controller and load it with any available configuration file (stored locally or off the network). Each controller may also be wiped by loading a special "blank" configuration file, ensuring the card is unable to transmit or receive messages. It is considered good practice to perform this operation before loading a new schedule, as it prevents the accidental reuse of prior configurations. A second version of the loader application was developed for use with single board computers and embedded devices only accessible through a serial interface. It uses a variation of the Newt library to render a keyboard driven text-based user interface within a terminal emulator.

A third program was developed to monitor and graphically display the flow of all critical traffic (TT or RC) within a TTEthernet network. Network monitoring in conventional Ethernet systems is usually accomplished on a switch-by-switch basis via a combination of switch port mirroring and specialized packet capture hardware/software. Monitoring traffic throughout an entire network generally requires more elaborate and expensive solutions. Alternatively, monitoring of all critical traffic within a TTEthernet network can be accomplished from a single listener device by taking advantage of the scheduling process. Specifically, the schedule can be adjusted such that the listener node is a recipient of each TT or RC message sent in the network (added to every VL). This monitoring technique requires no software changes to any of the devices in the network we intend to monitor - only new schedules. The monitoring application was built in two halves. The backend, written in $\mathrm{C}$, continuously reads critical traffic from any configurable number of virtual links. The customizable front end, written in Python, allows users to position tiles (representing devices) on an isometric grid and connect them based on the network architecture. The back end periodically updates the front end to display the flow of critical TTEthernet messages in real time.

Lastly, a text-based user interface (TUI) was built to exhibit flight computer synchronization in the AA2 flight test demonstration. The application (written in $\mathrm{C}$ and using the Ncurses terminal control library) runs locally on each flight computer and communicates directly with CFS to show the execution of the flight software in real time. The program uses a columned table layout to continuously display each CFS slot as it is processed, along with the applications woken by the CFS scheduler within that slot. For the AA2 flight test, the applications running on each redundant flight computer were therefore shown executing at the same time. The first slot in the schedule was highlighted to make this synchronization more apparent.

18 of 23

American Institute of Aeronautics and Astronautics 
The program uses a graphical icon to show the role of each flight computer within the set (i.e. master or slave). When failover occurs, the role displayed for each machine changes according to the priority levels of the remaining computers.

\section{VIII.G. Final Integrated Configurations for ARM and AA2 Missions}

The integrated setup used for the TTEthernet failover demonstration in the IPAS facility for the A\&S project's September FY14 Integrated Test (simulating the ARM mission) is depicted in Figure 7 and Figure 8. The closed TTEthernet-based flight control loop was located in Bay 1 of the IPAS testbed. It consisted of two redundant failover-capable flight computers and the corresponding Trick simulation machine. Communication within the control loop occurred via virtual links VL1, VL2, VL3, and VL4 (used for heartbeat-based failover only). A separate machine running Engineering DOUG Graphics Environment (EDGE) software received data from the Trick simulation and displayed a high fidelity 3D representation of the mission as it progressed.

To show the versatility of the TTEthernet-based failover method, dissimilar processors were used in the redundant flight computer set. One flight computer was realized on a standard Linux PC running CentOS 6.5 64 bit. A much more flight-like platform, the Space Micro Proton400k-L, acted as the second flight computer. The Proton400k-L incorporates a Freescale dual-core PowerPC processor in a radiation hardened $3 \mathrm{U}$ single board computer (SBC) platform. Of particular interest is its use of Time-Triple Modular Redundancy (TTMR) technology, designed to detect and correct single event upsets. ${ }^{37}$ To lower development time, the Proton 400k-L ran a 32-bit Linux variant instead of a traditional RTOS. The TTEthernet Linux drivers were adapted for use on this new platform.

Redundancy was present not just between the flight computers, but also within the TTEthernet network itself. Two parallel switches provided redundant pathways between the flight computers and the simulation machine. Each TTEthernet controller was physically wired to the same port on both switches. The network was configured such that transmissions of all time-triggered messages from each end system occurred over two parallel Ethernet cables. From the perspective of the flight software applications, only one virtual link was needed to communicate over both redundant channels. If no failures occurred, then all replicated traffic reached its intended receiver successfully. TTEthernet's built-in redundancy management transparently detected and discarded these duplicated frames. This capability ensured that if one switch was lost, the flow of critical traffic within the network would continue uninterrupted.

A computer running the network monitor software was connected to both TTEthernet switches and continuously displayed the flow of time-triggered messages within the control loop. The behavior of this interface depended on the failover method employed. In both cases, the display showed each computer receiving simulation data. Failure was induced by cutting power to the primary flight computer. If the heartbeat-based method was used, the display updated in real time as the primary computer ceased all transmission and the backup computer took over. If the command cycle approach was taken instead, icons on the screen changed color to indicate which computer was in the primary role.

The NetDev driver allowed the flight computers to communicate with other vehicle subsystems using standard Ethernet interfaces. The active flight computer outputted mission status information encapsulated in UDP datagrams. This data was transmitted in classical Ethernet frames through the TTEthernet switches, into the Bay 1 network, and to a touchscreen crew display. Additionally, an ECLSS simulator in Bay 2 of the IPAS facility broadcasted data such as partial pressure and ambient temperature to both flight computers. This data was relayed as UDP traffic over the standard Ethernet backbone, through a router connecting the Bay 2 and Bay 1 networks, and into the TTEthernet switches. All UDP traffic traveled through the same physical cabling as the time-triggered messages in the flight control loop.

Figure 9 and Figure 10 show the TTEthernet failover architecture used in the AA2 flight test during the A\&S project's May FY15 Integrated Test. Three redundant flight computers were realized using Linux PCs running CentOS 6.564 bit. Virtual links VL1, VL11, VL12, and VL13 were used to carry timetriggered traffic between the simulation and the flight computers. Unlike in the ARM demonstration, all communication within this control loop utilized the UDP and IPv4 transport and network layers on top of the time-triggered Ethernet data link layer. Synchronization between the flight computers was accomplished using rate-constrained traffic on virtual links VL21, VL22, and VL23. Again, redundant switches were used to increase fault-tolerance within the data network. Because the focus of the AA2 flight test was on implementing synchronization and improving the performance of the failover procedure, no best-effort communication was used to interface the flight computers with other vehicle subsystems. 


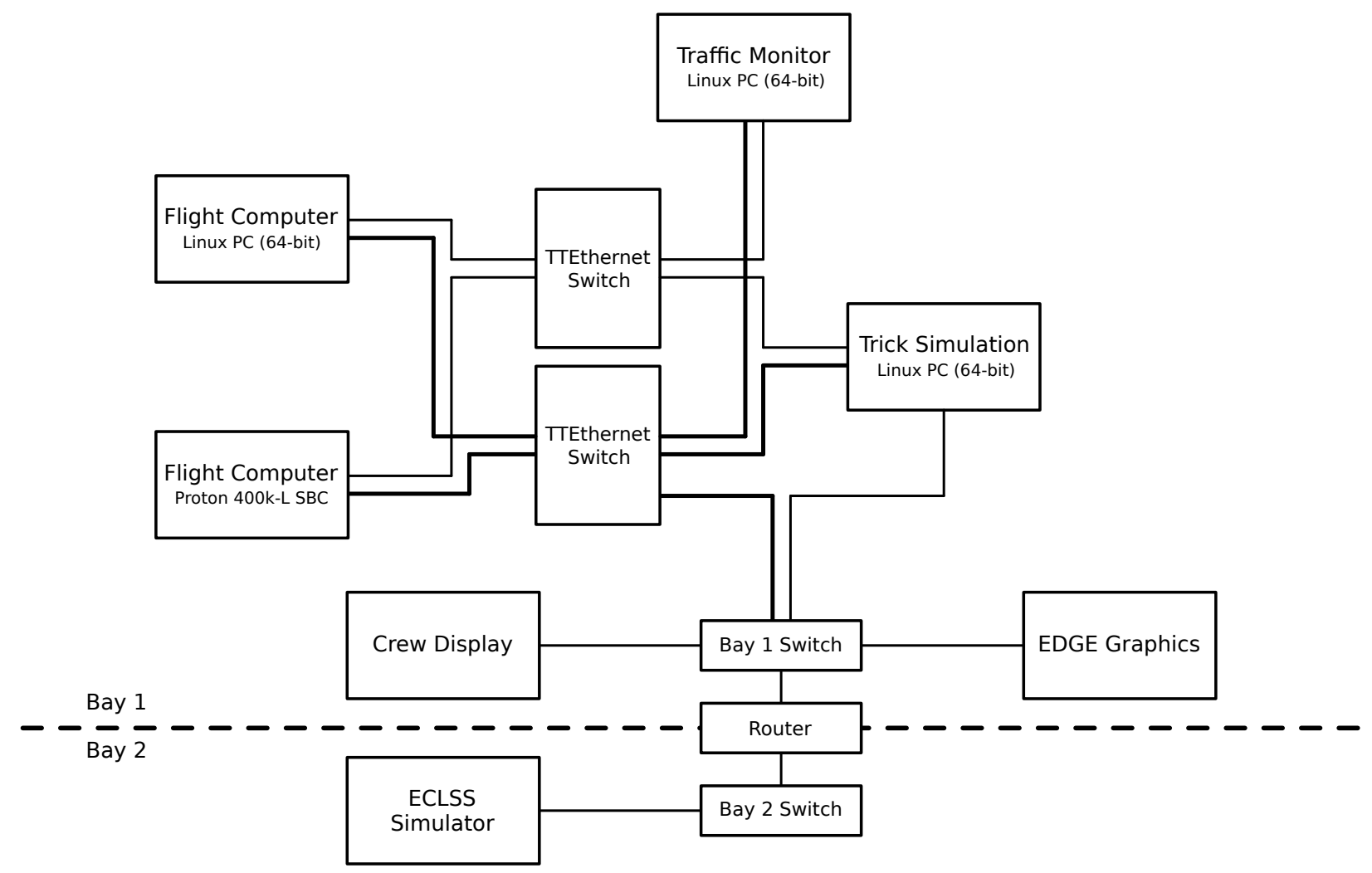

Figure 7. Redundant flight computer architecture for simulated Asteroid Redirect Mission (ARM) [physical links].

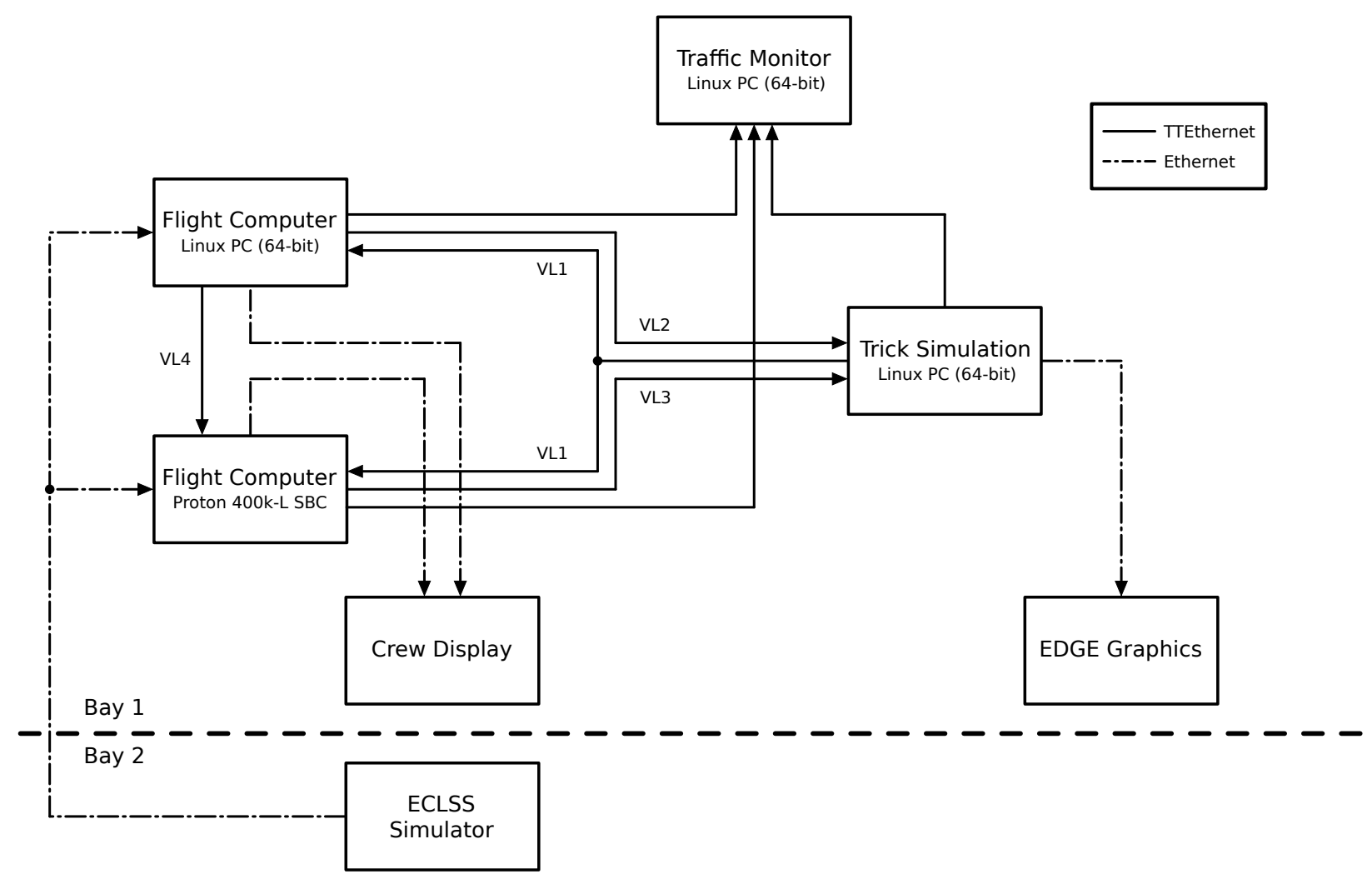

Figure 8. Redundant flight computer architecture for simulated Asteroid Redirect Mission (ARM) [data flow]. 


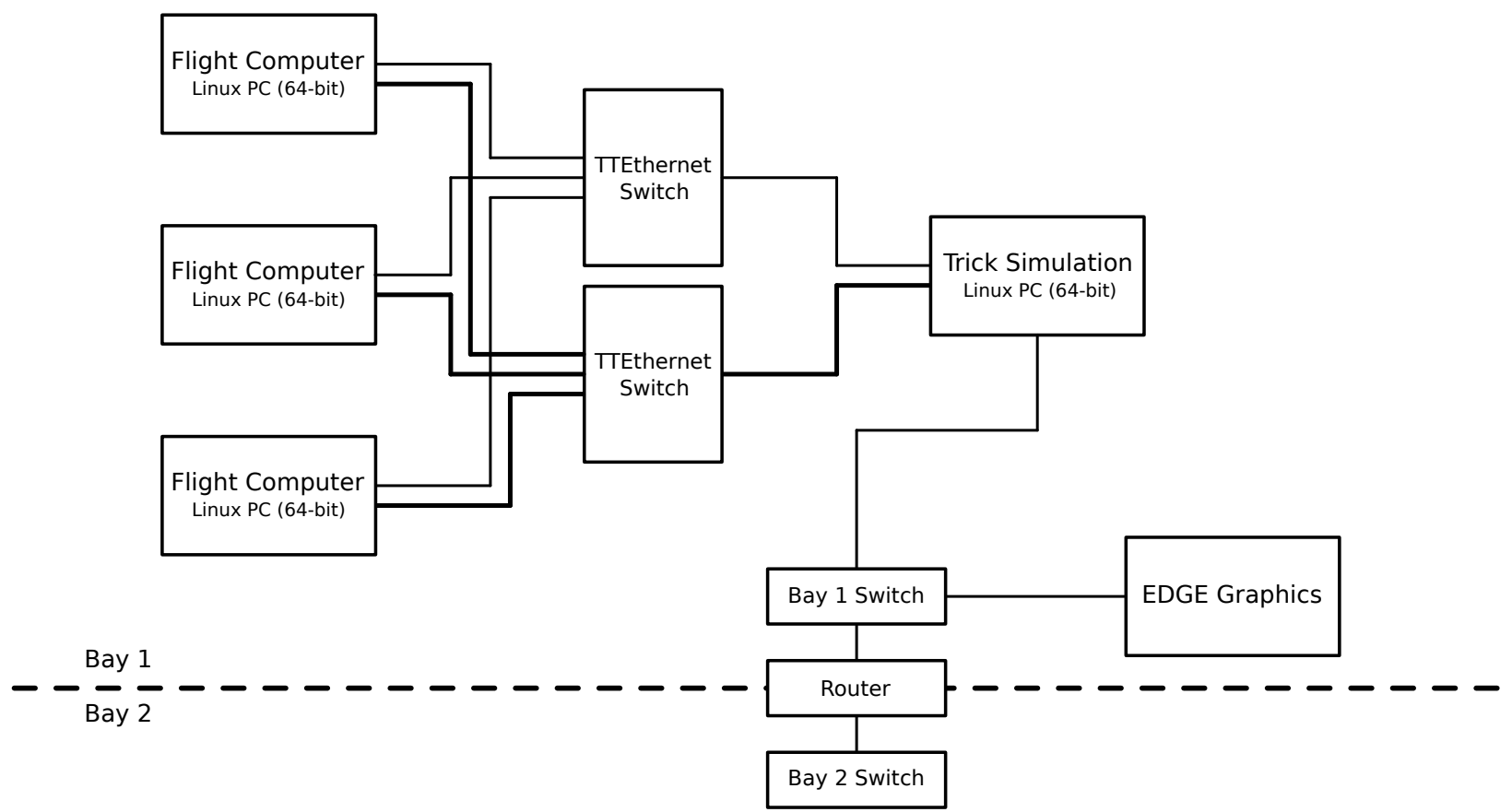

Figure 9. Redundant flight computer architecture for simulated Ascent Abort 2 (AA2) flight test [physical links].

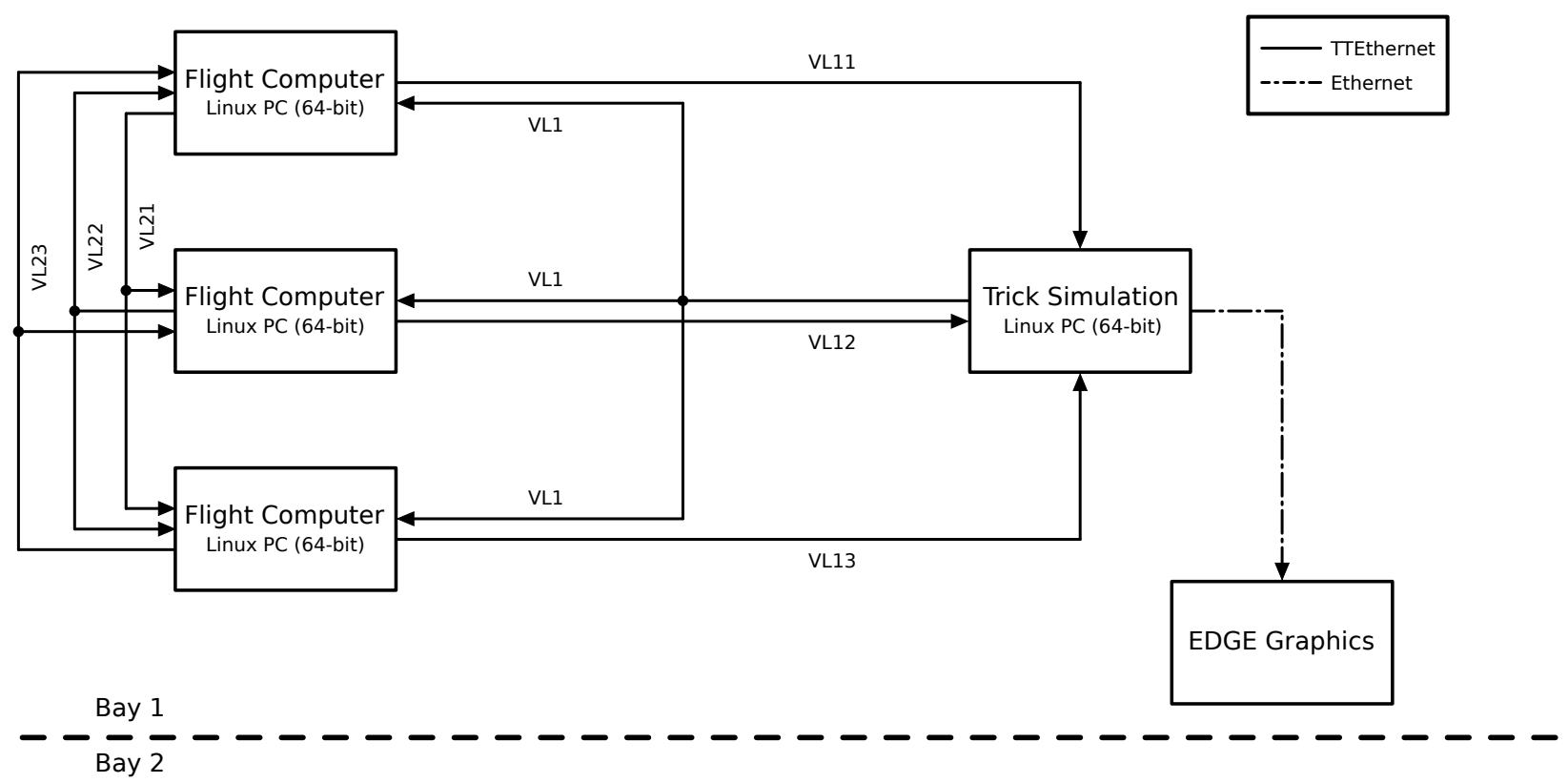

Figure 10. Redundant flight computer architecture for simulated Ascent Abort 2 (AA2) flight test [data flow]. 


\section{Future Work}

Future efforts will focus on increasing the fidelity of the fault-tolerant flight computer architecture and control loop. The failover configuration used for the AA2 flight test does not allow previously failed flight computers to rejoin the set. This limitation results from the design of the flight software, which retains state information over the course of the simulated mission. Future iterations of the failover architecture will likely incorporate the sharing of this data, allowing a new/rebooted flight computer to obtain this information from the other computers in the set. Moreover, future versions will likely have all flight computers (both the slaves and masters) broadcast their "current" CFS slot information. Slave computers will therefore be capable of detecting the failure of other slave computers of higher priority. This means that if the master computer dies, and the next highest priority computer is already dead, the third priority machine could take over control of the vehicle after one timeout period instead of two (the second timeout currently being used to discover the death of the second priority machine). Additionally, synchronization between computers could be accomplished only via the occurrence of cluster cycle interrupts on each machine, rather than relying on a master/slave commanding approach. Because the cluster cycle is a property of the entire TTEthernet synchronization domain, the start of a new cluster cycle is the same for every machine. On a real-time operating system (RTOS), the cluster cycle interrupt will occur simultaneously on all flight computers. The TTEthernet schedule can be developed such that all machines (flight computers and simulation) must be configured with the communication schedule (i.e. loaded with their respective configuration file generated by the networking planning tools) before network synchronization can occur. The process of loading this configuration file on each flight computer could then be incorporated into the startup procedures of the flight software. Once synchronization is established, an interrupt can be generated simultaneously on all flight computers - commencing execution of the CFS scheduler on each machine (and thus starting the count of cluster cycle interrupts). Use of precise network interrupts to drive flight software execution could prove an optimal solution for synchronization and vehicle timing in future spacecraft architectures.

TTEthernet's many advantages over standard Ethernet technology also make it attractive for inclusion in other projects. Moving forward, the TTEthernet-based failover work described in this paper will be combined with another A\&S project - the JSC Spacecraft Software Engineering Branch's standard Ethernet-based quad voting flight computer framework. The integration of these two projects is a vital step towards the A\&S project's goal of realizing a flexible mission agnostic spacecraft architecture. Furthermore, the A\&S audio group will investigate the use of $\mathrm{BE}$ and $\mathrm{RC}$ traffic to transport audio within a TTEthernet-based spacecraft network. The group will also explore methods for controlling the audio equipment remotely from the flight computers. The goal of this work will be to evaluate and guide the approach taken for implementing onboard communication systems on the NASA Orion vehicle.

\section{Acknowledgment}

The author would like to thank Lisa Erickson (JSC EC6), Daniel Benbenek (JSC EV2), and Adam Rawlin (JSC EV2) for their assistance in reviewing this paper.

\section{References}

\footnotetext{
${ }^{1}$ Rushby, J., "Partitioning in Avionics Architectures: Requirements, Mechanisms, and Assurance," Tech. rep., NASA Langley Research Center, Hampton, VA, June 1999.

${ }^{2}$ Driscoll, K., "Integrated Modular Avionics (IMA) Requirements and Development," Proc. ARTIST2 Workshop on Integrated Modular Avionics, Rome, Italy, Nov. 2007.

${ }^{3}$ Alena, R., Ossenfort, J., Laws, K., Goforth, A., and Figueroa, F., "Communications for Integrated Modular Avionics," Proc. Aerospace Conference, 2007 IEEE, March 2007.

${ }^{4}$ deLong, C., "MIL-STD-1553B Digital Time Division Command/Response Multiplex Data Bus," Industrial Communication Technology Handbook, Boca Raton, FL, 2nd ed., 2015.

${ }^{5}$ Gwaltney, D. A. and Briscoe, J. M., "Comparison of Communication Architectures for Spacecraft Modular Avionics Systems," Tech. rep., NASA Marshall Space Flight Center, Huntsville, AL, June 2006.

${ }^{6}$ Goforth, M., Ratliff, J., Hames, K., and Vitalpur, S., "Avionics Architectures for Exploration: Building a Better Approach for (Human) Spaceflight Avionics," Proc. SpaceOps 2014 International Conference on Space Operations, Pasadena, CA, May 2014.

7 “Aircraft Data Network, Part 7: Avionics Full Duplex Switched Ethernet (AFDX) Network," Sept. 2009.

${ }^{8}$ Steiner, W., Bauer, G., Hall, B., and Paulitsch, M., "Time-Triggered Ethernet," Time-Triggered Communication, Aug. 2015.
} 
${ }^{9}$ Crillo, W., Goodliff, K., Aaseng, G., Stromgren, C., and Maxwell, A., "Supportability for Beyond Low Earth Orbit Missions," Proc. AIAA SPACE 2011 Conference, Long Beach, CA, Sept. 2011.

${ }^{10}$ for Aeronautic, A. I. and Astronautics, editors, Guide for Estimating and Budgeting Weight and Power Contingencies, AIAA-G-020-1992.

${ }^{11}$ Bieber, P., Boniol, F., Boyer, M., Noulard, E., and Pagetti, C., "New Challenges for Future Avionics Architectures," 2014.

${ }^{12}$ Nguyen, Q., Yuknis, W., Pursley, S., Haghani, N., and Albaijes, D., "A High Performance Command and Data Handling System for NASA's Lunar Reconnaissance Orbiter," Proc. AIAA SPACE 2008 Conference, San Diego, CA, Sept. 2008.

${ }^{13}$ Webb, E., "Ethernet for Space Flight Applications," Proc. Aerospace Conference, 2002 IEEE, San Diego, CA, Jan. 2002.

${ }^{14}$ Dimitrakopoulos, G., "Fast Arbiters for On-Chip Network Switches," Proc. Computer Design, 2008 IEEE, Lake Tahoe, CA, Oct. 2008.

${ }^{15}$ Athanas, P., Pnevmatikatos, D., and Sklavos, N., Embedded Systems Design with FPGAs, Springer New York, 2013.

${ }^{16}$ Elhanany, I. and Hamdi, M., High-performance Packet Switching Architectures, Springer, 2007.

${ }^{17}$ Keslassy, I. and McKeown, N., "Maintaining Packet Order in Two-Stage Switches," Proc. Infocom, IEEE 2002, New York, June 2002.

${ }^{18}$ McKeown, N., "Fast Switched Backplane for a Gigabit Switched Router," Business Communications Review, Dec. 1997.

${ }^{19}$ Paulitsch, M. and Driscoll, K., "SAFEbus," Industrial Communication Technology Handbook, Boca Raton, FL, 2nd ed., 2015.

${ }^{20}$ Steiner, W., Bonomi, F., and Kopetz, H., "Towards synchronous deterministic channels for the Internet of Things," Proc. Internet of Things (WF-IoT), 2014 IEEE, Seoul, Republic of Korea, March 2014.

${ }^{21}$ Fuchs, C., "The Evolution of Avionics Networks From ARINC 429 to AFDX," Proc. Seminar Aerospace Networks SS2012, Munich, Germany, Aug. 2012.

${ }^{22}$ Steiner, W. and Paulitsch, M., "Time-Triggered Ethernet," Industrial Communication Technology Handbook, Boca Raton, FL, 2nd ed., 2015.

${ }^{23}$ Matzol, E., Ethernet in Automotive Networks, Master's thesis, KTH Royal Institute of Technology, Stockholm, Sweden, 2011.

24 "Time-Triggered Ethernet," Nov. 2011.

${ }^{25}$ Budhiraja, N. and Marzullo, K., "Tradeoffs in Implementing Primary-Backup Protocols," Proc. Symposium on Parallal and Distributed Processing, IEEE 1992, Oct. 1995.

${ }^{26}$ Newhouse, M., Friberg, K., Fesq, L., and Barley, B., "Fault Management Guiding Principles," Proc. Infotech @ Aerospace 2011 Conference: Unleashing Unmanned Systems, St. Louis, MI, March 2011.

${ }^{27}$ Hanaway, J. and Moorehead, R., Space Shuttle Avionics System, National Aeronautics and Space Administration, 1989.

${ }^{28}$ Othon, W., "iPAS: AES Flight System Technology Maturation for Human Spaceflight," Proc. SpaceOps 2014, Pasadena, CA, May 2014.

${ }^{29}$ Acevedo, A., Berndt, J., Othon, W., Arnold, J., and Gay, R., "ANTARES: Spacecraft Simulation for Multiple User Communities and Facilities," Tech. rep., NASA Johnson Space Center, Houston, TX, Aug. 2007.

30 "Core Flight System (CFS): Development Standards Document," July 2011.

${ }^{31}$ Wildermann, C., "cFE/CFS (Core Flight Executive/Core Flight System)," Proc. Flight Software Workshop 2008, Laurel, MD, Nov. 2008.

${ }^{32}$ Ganesan, D., Lindvall, M., McComas, D., Bartholomew, M., Slegel, S., and Medina, B., "Architecture-Based Unit Testing of the Flight Software Product Line," Proc. 14th International Software Product Line Conference (SPLC), Jeju Island, Republic of Korea, Sept. 2010.

${ }^{33}$ Wilmot, J., "A Reusable and Adaptable Software Architecture for Embedded Space Flight System: The Core Flight Software System (CFS)," Proc. International Conference on Hardware/Software Codesign and System Synthesis, Jersey City, NJ, Sept. 2005.

${ }^{34}$ Postel, J., "Internet Protocol," RFC 791 (INTERNET STANDARD), Sept. 1981, Updated by RFCs 1349, $2474,6864$.

${ }^{35}$ Postel, J., "User Datagram Protocol," RFC 768 (INTERNET STANDARD), Aug. 1980.

36 "TTE Plan: The TTEthernet Scheduler," Sept. 2013.

${ }^{37}$ Czajkowski, D., "Rad Hard High Performance Computing," May 2008. 